\title{
Alternative splicing promotes tumour aggressiveness and drug resistance in African American prostate cancer
}

\author{
Bi-Dar Wang ${ }^{1,2}$, Kristin Ceniccola ${ }^{1}$, SuJin Hwang ${ }^{3}$, Ramez Andrawis ${ }^{4}$, Anelia Horvath ${ }^{1}$, Jennifer A. Freedman ${ }^{5}$, \\ Jacqueline Olender ${ }^{1}$, Stefan Knapp ${ }^{6,7}$, Travers Ching ${ }^{8}$, Lana Garmire ${ }^{8}$, Vyomesh Patel ${ }^{9}$, \\ Mariano A. Garcia-Blanco ${ }^{10}$, Steven R. Patierno ${ }^{5}$ \& Norman H. Lee ${ }^{1}$
}

Clinical challenges exist in reducing prostate cancer ( $\mathrm{PCa}$ ) disparities. The RNA splicing landscape of $\mathrm{PCa}$ across racial populations has not been fully explored as a potential molecular mechanism contributing to race-related tumour aggressiveness. Here, we identify novel genome-wide, race-specific RNA splicing events as critical drivers of PCa aggressiveness and therapeutic resistance in African American (AA) men. AA-enriched splice variants of PIK3CD, FGFR3, TSC2 and RASGRP2 contribute to greater oncogenic potential compared with corresponding European American (EA)-expressing variants. Ectopic overexpression of the newly cloned AA-enriched variant, PIK3CD-S, in EA PCa cell lines enhances AKT/mTOR signalling and increases proliferative and invasive capacity in vitro and confers resistance to selective PI3K $\delta$ inhibitor, CAL-101 (idelalisib), in mouse xenograft models. High PIK3CD-S expression in $\mathrm{PCa}$ specimens associates with poor survival. These results highlight the potential of RNA splice variants to serve as novel biomarkers and molecular targets for developmental therapeutics in aggressive PCa.

\footnotetext{
${ }^{1}$ Department of Pharmacology and Physiology, School of Medicine and Health Sciences, The George Washington University, Washington, District Of Columbia 20037, USA. ${ }^{2}$ Department of Pharmaceutical Sciences, School of Pharmacy and Health Professions, University of Maryland Eastern Shore, Princess Anne, Maryland 21853, USA. ${ }^{3}$ Department of Microbiology, Immunology and Tropical Medicine, School of Medicine and Health Sciences, The George Washington University, Washington, District Of Columbia 20037, USA. ${ }^{4}$ Department of Urology, School of Medicine and Health Sciences, The George Washington University, Washington, District Of Columbia 20037, USA. ${ }^{5}$ Duke Cancer Institute and Department of Medicine, Duke University Medical Center, Durham, North Carolina 27710, USA. ${ }^{6}$ Department of Clinical Pharmacology, University of Oxford, Oxford OX3 7BN, UK. ${ }^{7}$ The Nuffield Department of Clinical Medicine, Structural Genomics Consortium, University of Oxford, Oxford OX3 7BN, UK. ${ }^{8}$ Cancer Epidemiology Program, University of Hawaii Cancer Center, Honolulu, Hawaii 96813, USA. ${ }^{9}$ Oral and Pharyngeal Cancer Branch, National Institute of Dental and Craniofacial Research, National Institutes of Health, Bethesda, Maryland 20892, USA. ${ }^{10}$ Department of Biochemistry \& Molecular Biology, The University of Texas Medical Branch at Galveston, Galveston, Texas 77555, USA. Correspondence and requests for materials should be addressed to N.H.L. (email: nhlee@gwu.edu).
} 
$\mathrm{P}$ rostate cancer (PCa) is the most commonly diagnosed cancer and the second leading cause of cancer death among American men ${ }^{1}$. Striking population disparities in PCa risk and clinical outcome have been observed across racial groups. Notably, African American (AA) men exhibit 1.6-fold higher incidence and 2.4-fold higher mortality rates of PCa compared with European American (EA) men ${ }^{2,3}$. Socioeconomic factors remain a major component accounting for the PCa disparities

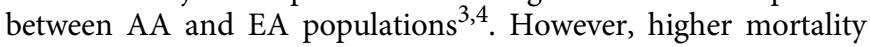
and recurrence rates are still observed in AA PCa even after adjustment of socioeconomic factors ${ }^{5}$, suggesting that intrinsic biological differences also play a contributing role in $\mathrm{PCa}$ disparities $^{6-10}$.

Alternative splicing (AS) is a post-transcriptional process allowing for the generation of alternative mRNA transcripts that encode structurally and functionally disparate protein isoforms. Next-generation sequencing suggests that $>90 \%$ of human genes undergo $\mathrm{AS}^{11}$, and the resulting complexity in the transcriptome explains how $\sim 20,000$ protein-coding genes in the genome can lead to $>250,000$ distinct proteins in the proteome. Accumulating evidence indicates that alternative and/or aberrant splicing of precursor (pre)-mRNA plays an important but largely underappreciated role in cancers ${ }^{12-15}$, including $\mathrm{PCa}^{16}$. For example, the B-cell lymphoma 2-like 1 (BCL2L1) pre-mRNA is alternatively spliced into two variants, $B c l-x S$ and $B c l-x L$, encoding protein isoforms with opposite biological effects $^{17}$. Bcl-xS is a pro-apoptotic protein, while BcL-xL has anti-apoptotic properties conferring chemotherapy resistance in PCa cell line PC-3 (ref. 15) as well as castration-resistant xenograft growth ${ }^{18}$. Manipulation of splicing to decrease Bcl-xL and increase Bcl-xS levels has been shown to reduce tumour load ${ }^{19}$. Fibroblast growth factor receptor 2 (FGFR2) pre-mRNA also undergoes AS, where FGFR2-IIIb is predominately expressed in epithelial cells and FGFR2-IIIc is primarily associated with

a

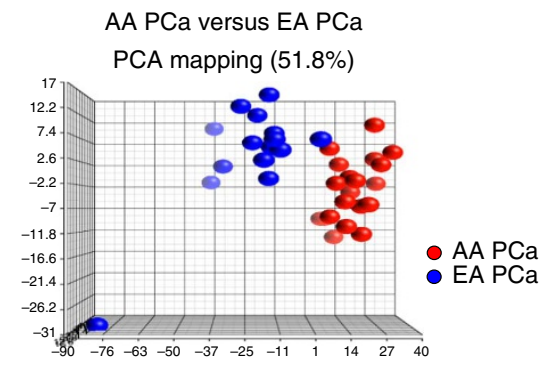

b

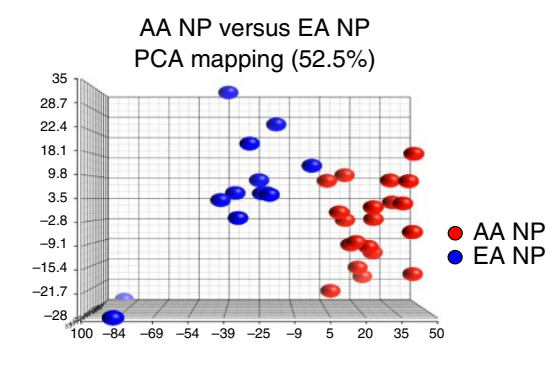

AA NP versus EA NP

\section{C}

Venn diagrams

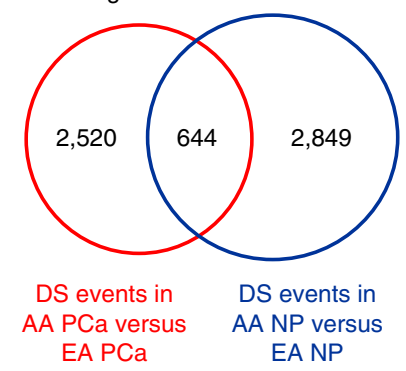

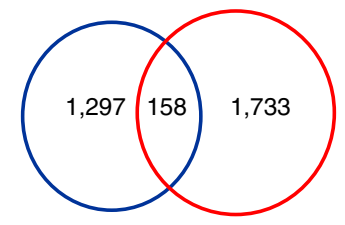

DS events in DS events in EA PCa versus AA PCa versus EA NP
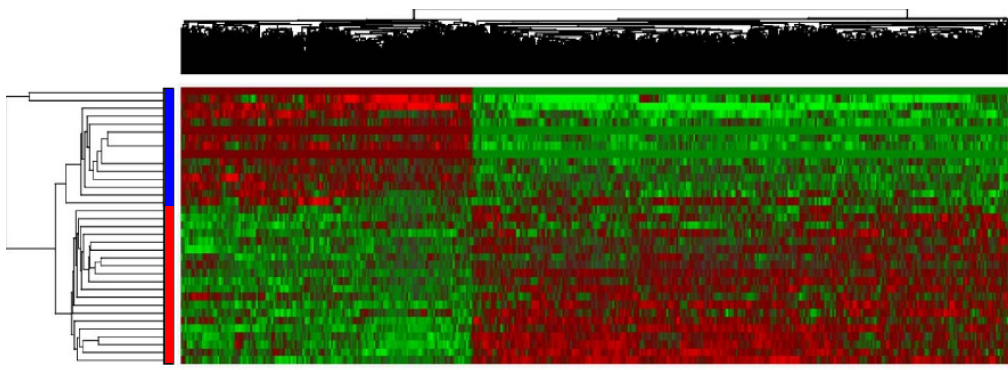
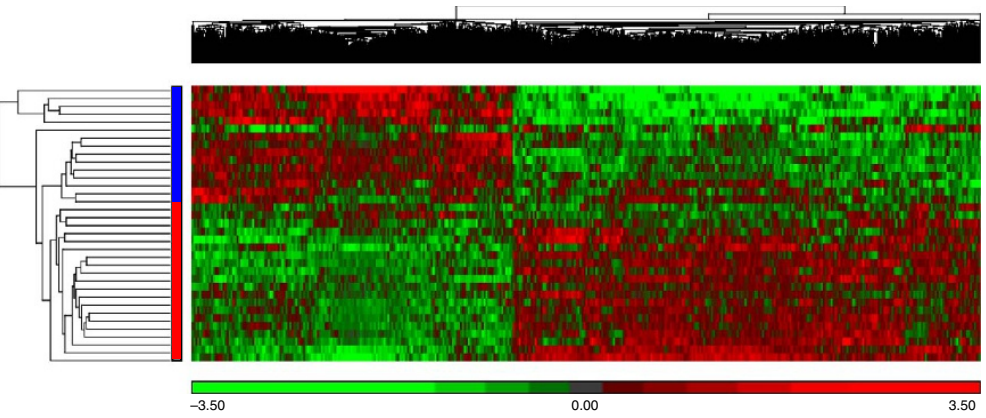

$-4.03$

0.00 4.03

Figure 1 | Differential alternative splicing events in AA PCa compared with EA PCa and AA NP compared with EA NP specimens. (a) Principal component analysis (PCA) plot and two-dimensional (2D) clustergram depicting 3,112 significant differentially expressed exons in 20 independent AA PCa versus 15 independent EA PCa specimens. (b) PCA plot and 2D clustergram depicting 3,384 significant differentially expressed exons in 20 AA NP versus 15 EA NP specimens. AA and EA specimens are represented by red and blue circles/bars, respectively. Rows represent specimens and columns represent exons in hierarchical clustergrams. Log2 expression values of exons were subjected to 2D unsupervised hierarchical clustering using average linkage method and Euclidean distance. (c) Venn diagrams of DS events in AA PCa versus EA PCa and AA NP versus EA NP, DS events in EA PCa versus EA NP and AA PCa versus AA NP and differentially expressed genes in AA PCa versus EA PCa and DS events in AA PCa versus EA PCa. (d) A majority of the genes with DS events in AA PCa versus EA PCa were functionally associated with cancer. The top three 'other diseases' were gastrointestinal disease, organismal injury and abnormalities and reproductive system disease. 
epithelial-to-mesenchymal transition of $\mathrm{PCa}$ cells $^{20}$. Another example is the TMPRSS2-ERG gene fusion commonly found in $\mathrm{PCa}$ and associated with poor clinical outcome ${ }^{21,22}$. In a comparative study of TMPRSS2-ERG variants ectopically overexpressed in prostatic epithelial cells, variants containing a 72 base exon $(+72)$ mediate increased cell proliferation and invasion ${ }^{14}$. Androgen receptor (AR) signalling is critically associated with $\mathrm{PCa}$ growth $^{23}$ and splice variant $A R-V 7$ is overexpressed in hormone-refractory $\mathrm{PCa}$, being correlated with poor patient survival and higher recurrence rates ${ }^{24,25}$.

Despite the significance of alternative/aberrant splicing in $\mathrm{PCa}$ progression irrespective of race, the occurrence of racespecific/-enriched $\mathrm{PCa}$ splicing events and a causal relationship between these events and observed PCa disparities remains unexplored. For example, it is unclear whether FGFR2-IIIc, TMPRSS2-ERG + 72, $A R-V 7$ and/or other as yet undiscovered variants associated with more aggressive $\mathrm{PCa}$ might be predominantly or selectively expressed in AA PCa, thus contributing to PCa disparities. In addition, it is unknown whether differences in mRNA splicing along racial/population lines occur in only a limited number of genes or more globally across the transcriptome. If the latter, it will be important to ascertain whether these genome-wide, differential splicing (DS) events are overrepresented within specific gene ontologies (that is, proto-oncogenes, tumour suppressor genes). Lastly, assessment of the functional consequences of any race-specific (or enriched) splicing events will provide critical further insight into the genetic/molecular mechanisms underlying PCa disparities. To this end, we have applied a functional genomics approach to address these questions. Our results underscore the leveraging of population differences in tumour biology to discover novel splice variants that will likely serve as novel biomarkers and/or molecular targets for developmental therapeutics against aggressive AA PCa, identify previously hidden splice variants encoding oncogenic signalling proteins resistant to small-molecule inhibitors (SMIs), and assimilate splice variant information for prognostication of cancer aggressiveness and/or therapeutic responsiveness.

\section{Results}

Genome-wide DS events in AA versus EA PCa. A total of $35 \mathrm{PCa}(20 \mathrm{AA} / 15 \mathrm{EA})$ and 35 patient-matched normal prostate (NP) specimens (20 AA/15 EA) derived from chemo-/hormone-/ radiation-naive patients were interrogated using the Affymetrix Human Exon 1.0 ST GeneChip to assess DS events. Gleason scores of PCa specimens (range 6-8) and patient ages (range 49-81 years) were not significantly different between AA and EA cohorts $(P>0.05$, Fisher's exact test). In AA PCa versus EA PCa and AA NP versus EA NP, the significant differentially expressed exons (Fig. 1a,b) could be modelled using the AS analysis of variance (ANOVA) approach ${ }^{26}$ into 2,520 and 2,849 DS events, respectively (Supplementary Data 1 and Supplementary Fig. 1). As depicted in the Venn diagram (Fig. 1c), 1,876 genes (2,520 minus 644) exhibited DS events unique to AA PCa versus EA PCa, 2,205 differentially spliced genes (2,849-644) were unique to AA NP versus EA NP and 644 DS events were in common (that is, DS events preexisting in AA NP versus EA NP and preserved in AA PCa versus EA PCa). Examples of genes with preexisting DS events included PIK3CD, ITGA4 and MET, while RASGRP2, NF1 and BAK1 are examples of differentially spliced genes occurring only in AA PCa versus EA PCa. In EA PCa versus EA NP and AA PCa versus AA NP, the significant differentially expressed exons (Supplementary Fig. 2) could be modelled into 1,297 and 1,733 DS events, respectively (Fig. 1c). Presumably, a subset of 1,575 genes $(1,733-158)$ with DS events unique to AA PCa may contribute to PCa disparities. Examples in this category included FGFR3 and TSC2 (Supplementary Data 1). On the other hand, a subset of 158 genes with DS events in common to both $\mathrm{AA}$ and EA PCa may contribute to $\mathrm{PCa}$ progression regardless of race (Fig. 1c). Consistently, such genes included TMPRSS2 and AR (Supplementary Data 1c,d). Analysis of the exon array data employing both gene-wise $e^{9,10}$ and AS ANOVA modelling approaches ${ }^{26}$ identified 898 genes $(1,188-290)$ that were differentially expressed but not exhibiting DS in AA PCa versus EA PCa, and 2,230 $(2,520-290)$ genes undergoing DS but not differential expression (for example, level of variant ' $A$ ' for gene ' $\mathrm{X}$ ' in AA $\mathrm{PCa}$ equivalent to variant ' $\mathrm{B}$ ' for gene ' $\mathrm{X}$ ' in EA PCa; Fig. 1c).

Prevalence of DS events in cancer-associated pathways. We categorized genes undergoing DS in AA PCa versus EA PCa based on molecular function, gene ontology and disease association. Relevant cancer-related ontologies included cell growth and proliferation, cell death and survival, cellular movement, cell adhesion and DNA damage/repair $(P$ values ranged from $6.54 \times 10^{-12}$ to $1.88 \times 10^{-2}$, Fisher's exact test; Supplementary Data 2$)$. Notably, a large fraction (1,816 out of $2,520,71.8 \%)$ of the differentially spliced genes were discovered to be overrepresented across multiple cancers, including colorectal, renal, breast, brain, lung, stomach, prostate and haematologic cancers $\left(P\right.$ values ranged from $1.43 \times 10^{-9}$ to $1.96 \times 10^{-2}$, Fisher's exact test; Fig. 1d and Supplementary Data 2). There was an unexpected skewing in the distribution of in-frame versus out-of-frame exon skipping events in cancer-related genes, where in-frame events were significantly favoured in AA over EA PCa specimens $(P<0.05$, Fisher's exact test; Supplementary Table 1$)$. This finding was in line with an overall significant preference for in-frame events across all genes (cancer-related and noncancer-related) in AA PCa specimens (Supplementary Table 1). In the case of noncancer-related genes only, there was no significant skewing of in-frame distribution events between AA versus EA PCa (Supplementary Table 1).

We also examined the distribution of DS events across cell signalling pathways. There was a striking significant overrepresentation of DS events in multiple oncogenic signalling pathways, including epidermal growth factor (EGF), vascular endothelial growth factor (VEGF), phosphatase and tensin homolog (PTEN), phosphatidylinositol-3-kinase (PI3K)/AKT, extracellular signal-regulated kinase/mitogen-activated protein kinase (ERK/MAPK) and nuclear factor $-\kappa \mathrm{B}(\mathrm{NF}-\kappa \mathrm{B})$ signalling (Fisher's exact test, $P$ values ranged from 0.00126 to 0.02089 ; Supplementary Fig. 3 and Supplementary Data 3). Interestingly, many of these same pathways are known to be mutated based on earlier cancer genome sequencing studies ${ }^{27-29}$. A composite oncogenic signalling pathway comprising DS events found in AA PCa versus EA PCa is depicted in Fig. 2. Taken together, our data provide strong evidence that DS events may play a critical role in PCa disparities.

Validation of AS variants in AA versus EA PCa. We proceeded to validate a subset of both proto-oncogenes and tumour suppressor genes with DS events in our composite cancer signalling pathway, including PIK3CD, FGFR3, TSC2, ITGA4, MET, NF1, BAK1, ATM and RASGRP2 (Fig. 2). Real-time PCR (RT-PCR) was performed on RNA samples obtained from AA and EA PCa specimens originally interrogated by the exon arrays. Primer pairs or trios were designed for RT-PCR to amplify simultaneously multiple variants of each gene (Fig. 3a). As shown in Fig. 3b, AA PCa specimens contained both PIK3CD long (PIK3CD-L, including exon 20) and short (PIK3CD-S, missing exon 20) variants, whereas EA PCa samples predominately 


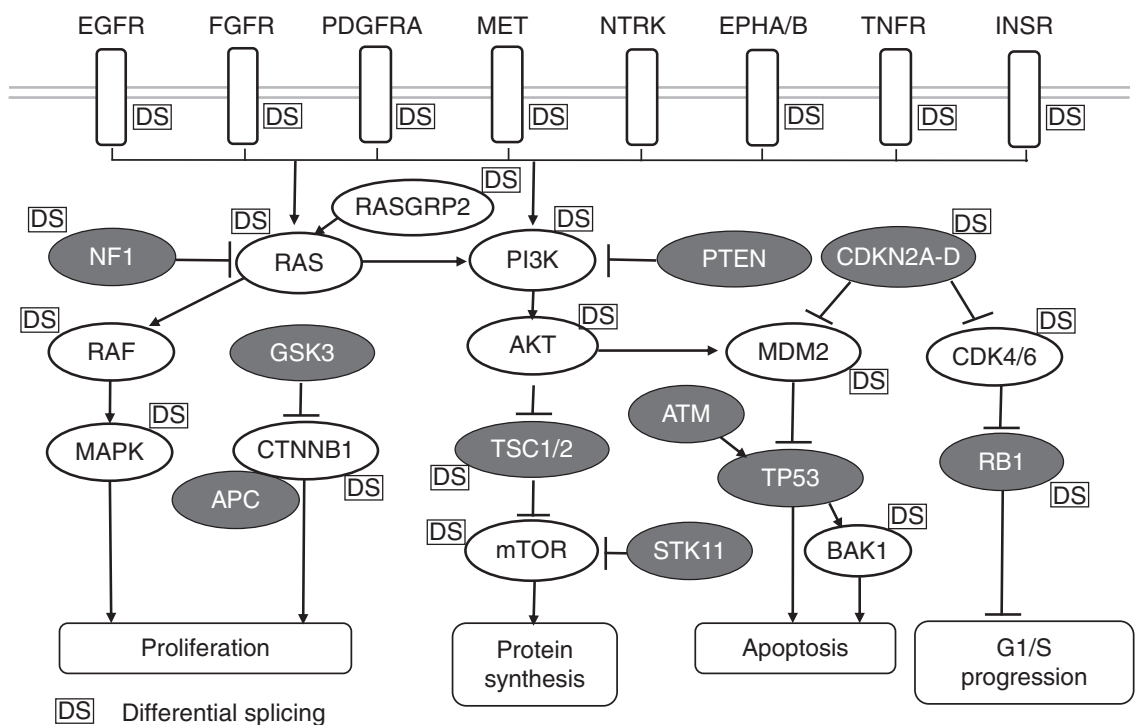

Figure 2 | Representation of differential splicing events in a composite oncogenic signalling pathway. DS events in AA PCa versus EA PCa were frequently detected in multiple oncogenic signalling pathways. Depicted pathway is a composite of PI3K/AKT/mTOR, RAS/RAF/MAPK, CDK/RB1, MDM2/TP53 and WNT/GSK3/CTNNB1/APC signalling. Open figures indicate oncogenes and closed figures indicate tumour suppressor genes. 'DS' indicates that a differential alternative splicing event was detected for a particular oncogene or tumour suppressor gene.

a

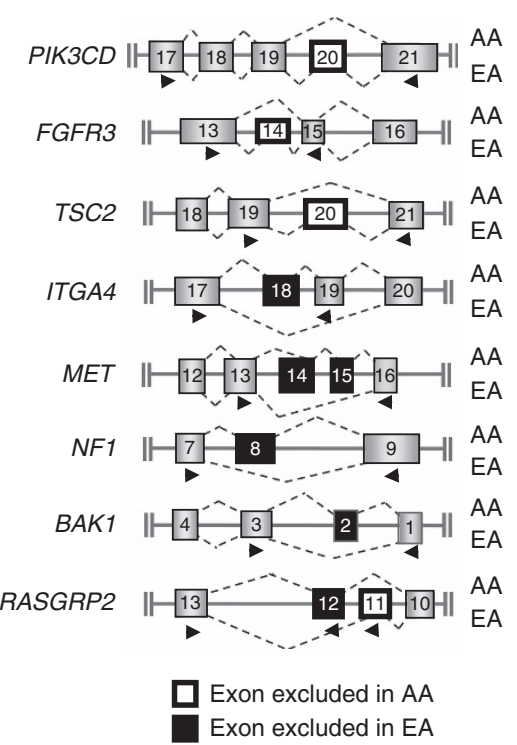

b

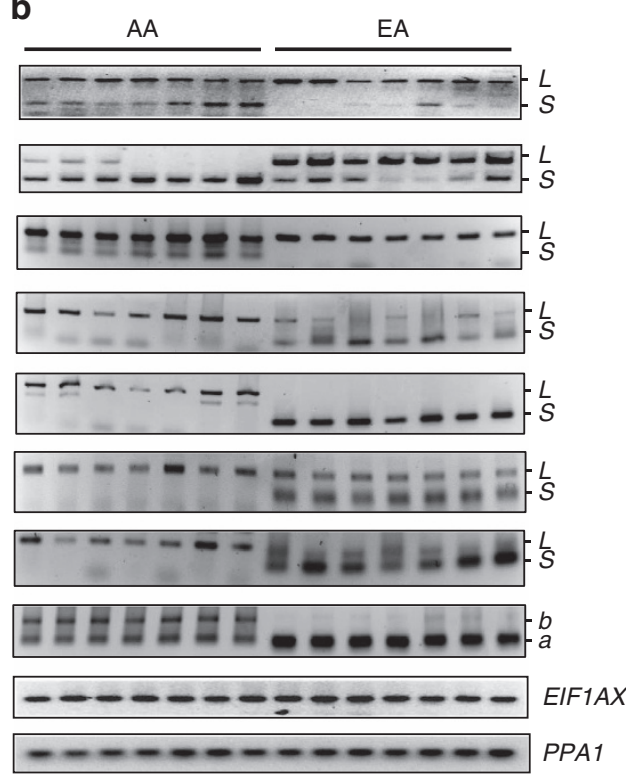

C

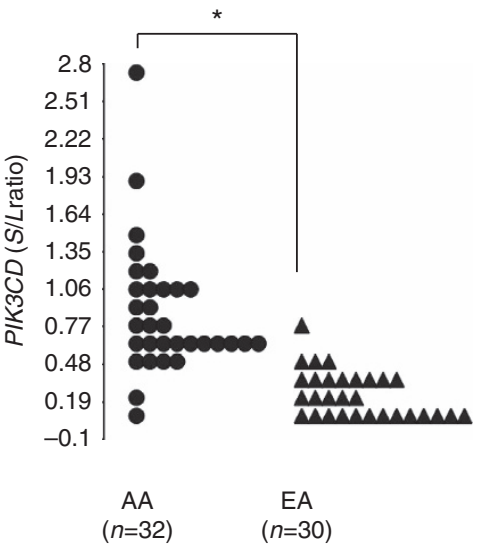

Figure 3 | Validation of differential alternative splicing of oncogenes and tumour suppressor genes in AA versus EA PCa specimens. (a) Schematic representation of DS events within the indicated genes in AA PCa (top dashed lines connecting exons) and EA PCa (bottom dashed lines) based on alternative splicing ANOVA model. Closed arrowheads below exons represent primer location in qRT-PCR validation of alternatively spliced transcripts. (b) Representative RT-PCR results validating race-specific/-enriched variant transcripts in either AA PCa or EA PCa specimens. Shown are the RT-PCR results for the AA-specific/-enriched variants PI3KCD-S, FGFR3-S, TSC2-S, ITGA4-L, MET-L, NF1-L, BAK1-L and RASGRP2-b; and EA-specific/-enriched variants PIK3CD-L, FGFR3-L, TSC2-L, ITGA4-S, MET-S, NF1-S, BAK1-S and RASGRP2-a. Each lane represents an RT-PCR result from an independent PCa specimen that was also interrogated in exon array experiments. RT-PCR of EIF1AX and PPA1 transcripts served as loading controls. The qRT-PCR results are summarized in Supplementary Fig. 4. Unprocessed RT-PCR images are shown in Supplementary Fig. 11. (c) Additional validation and quantification of the ratio of the AA-enriched PI3KCD-S (short) variant and the race-independent PI3KCD-L (long) variant in a separate cohort of PCa patient specimens. RNA was isolated from $n=32 \mathrm{AA} \mathrm{PCa}$ and $n=30$ EA PCa specimens and subjected to qRT-PCR. Shown is a plot of the ratio of S/L. EIF1AX and PPA1 transcripts served as internal normalization controls. ${ }^{\star} P<0.05$ using two-sided Student's $t$-test. Variance was similar among groups being compared.

expressed PIK3CD-L. Hence, the RT-PCR results were in agreement with the exon array data, indicating the presence of an AAenriched PIK3CD-S variant. Analogous findings were obtained where either a short or long variant of each gene was confirmed by RT-PCR to be enriched or uniquely expressed in AA (TSC2-S,
ITGA4-L, MET-L, BAK1-L) or EA PCa (FGFR3-L, ITGA4-S, MET-S, NF1-S, BAK1-S) (Fig. 3b; Supplementary Fig. 4 for quantitative RT-PCR (qRT-PCR) results from $n=22-25$ AA and $n=21-24$ EA PCa specimens). Exon array data also revealed two alternative RASGRP2 transcripts with apparent mutually 
exclusive exon skipping events (Fig. 3a and Supplementary Fig. 1d). RT-PCR validation likewise confirmed that a RASGRP2-b variant (exon 11 excluded) was exclusively expressed in AA PCa, while a RASGRP2- $a$ variant (exon 12 excluded) was enriched in EA PCa (Fig. $3 \mathrm{~b}$ and Supplementary Fig. 4). We were unable to validate DS of $A T M$, whereas two additional genes (GSK3A and EPHA1) identified by exon arrays as not undergoing DS were confirmed by RT-PCR. In summary, there was strong agreement $(10 / 11,91 \%)$ between exon array and RT-PCR results, thus providing an internal quality metric to our global DS analysis of $\mathrm{AA}$ and EA PCa (see Methods for additional metrics).

The race-dependent expression of PIK3CD variants was particularly interesting owing to recent findings implicating $\mathrm{PI} 3 \mathrm{~K} \delta(\mathrm{p} 110 \delta)$ kinase activity in haematologic malignancies as well as other cancer types ${ }^{30-33}$. In a separate cohort of PCa specimens obtained from 32 AAs (age range 52-76 years, Gleason score range 6-8) and 30 EAs (age range 50-82 years, Gleason score range 6-8; not significantly different from AA, Fisher's exact test, $P>0.05)$, quantitative RT-PCR validation was performed reaffirming significantly higher levels of PIK3CD-S relative to $P I K 3 C D-L$ in AA versus EA PCa specimens (Fig. $3 c$ ). Given the robustness and potential significance of these findings, subsequent in vitro and in vivo studies centred on the PIK3CD variants, as described below.

Molecular cloning of PIK3CD splice variants. The AA-enriched $P I K 3 C D-S$ variant has never before been described in the literature nor the UCSC (University of California, Santa Cruz) (genome.ucsc.edu) or Ensembl Genome Browser (www.ensembl.org). Consequently, we cloned the full-length versions of PIK3CD-S from AA PCa cell line MDA PCa $2 \mathrm{~b}$, and PIK3CD-L from MDA PCa $2 \mathrm{~b}$ as well as EA PCa cell lines VCaP and LNCaP using standard molecular approaches $\left(5^{\prime}\right.$ - and $3^{\prime}$-RACE (rapid amplification of cDNA ends) ${ }^{34}$ ). We likewise cloned matching PIK3CD-S and PIK3CD-L variants from PCa patient specimens. Supplementary Fig. 5 schematically depicts the full-length clones of PIK3CD-L (comprising a total of 24 exons) along with three different AA PIK3CD-S variants (variant excluding exon 8, exon 20 or both exons 8 and 20) and one AA large deletion variant of the PIK3CD gene. Interestingly, exclusion of exon 8 eliminates a 30 -amino acid segment situated between the Ras-binding and C2 domains, while exclusion of exon 20 deletes a 56-amino acid segment located in the catalytic domain of PI3K $\delta$. In subsequent functional studies involving ectopic overexpression of the short variant (see below), we concentrated our efforts on the variant missing exon 20 given the possibility that kinase activity may be affected.

PIK3CD $S$ isoform augments invasion and proliferation. We hypothesized that the splice variants specific or enriched in AA PCa may contribute to a more aggressive oncogenic phenotype. To test this, we designed exon-specific and exon junction-specific short interfering RNAs (siRNAs) to target PIK3CD- $L$ and PIK3CD-S, respectively, in EA and AA PCa cell lines and examined the functional consequences of these knockdowns on cell proliferation and invasion. A similar strategy was applied to investigate the biological significance of the variants of FGFR3, TSC2 and RASGRP2. VCaP and MDA PCa $2 \mathrm{~b}$ cells were used as population-specific PCa models, as these two cell lines represent bone metastases derived from castration-resistant EA and AA PCa patients, respectively ${ }^{35,36}$. Transfection of VCaP cells with exon 20-specific siRNA $\left(\mathrm{siP}_{20}\right)$ successfully knocked down $P I K 3 C D-L$ expression by $>8$-fold compared with nonsense siRNA (Fig. 4a, left panel), resulting in a significant loss of proliferative and invasive function in $\mathrm{VCaP}$ cells (Fig. 4b, left).
Conversely, in MDA PCa 2b cells, a >5-fold knockdown of $P I K 3 C D-L$ increased the ratio of PIK3CD-S/PIK3CD- $L$ expression by nearly twofold (Fig. 4a, right panel; $1.88 \mathrm{~S} / \mathrm{L}$ ratio for nonsense versus $3.46 \mathrm{~S} / \mathrm{L}$ ratio for $\mathrm{siP}_{20}$-transfected cells), and this 'enrichment' of AA-enriched PIK3CD-S subsequently enhanced proliferation and invasion of the AA cell line (Fig. 4a, right panel). Moreover, MDA PCa $2 b$ cells exhibited significantly higher basal invasive and proliferative capacities compared with VCaP cells (proliferation and invasion of siNS-transfected MDA PCa $2 \mathrm{~b}$ versus siNS-transfected VCaP; Fig. 4b, left and right panels). To further evaluate the functional impact of PIK3CD-S expression on cell proliferation and invasion, the EA and AA PCa cell lines were transfected with $\mathrm{siP}_{\mathrm{j}}$ (siRNA specifically targeting the junction of exons 19 and 21). Transfection of $\operatorname{siP}_{j}$ had no effect on VCaP proliferation and invasion, as expected since this EA line does not significantly express PIK3CD-S (Fig. 4a,b; right panels). On transfection of MDA PCa 2 b cells with $\mathrm{siP}_{\mathrm{j}}, P I K 3 C D-S$ expression was significantly knocked down (Fig. 4a, right), resulting in a loss of cell proliferation and invasion (Fig. 4b, right). Taken together, these results suggest that PIK3CD-S is the more aggressive variant, promoting $\mathrm{PCa}$ proliferation and invasion to a greater extent than PIK3CD-L.

Several additional exon-specific siRNAs were designed to test whether other AA-specific/-enriched splice variants also functionally contribute to greater $\mathrm{PCa}$ aggressiveness. SiRNAs targeting exon 14 (siFGFR3-ex14), exon 20 (siTSC2-ex20) and exon 11 (siRASGRP2-ex11) were used to selectively suppress expression of FGFR3- $L$, TSC2- $L$ and RASGRP2- $a$ variants (predominately expressed in EA), respectively. Upon siRNAmediated knockdown of FGFR3-L, TSC2- $L$ or RASGRP2-a (Supplementary Fig. 6a-c, top panels) in MDA PCa $2 \mathrm{~b}$ cells, the expression ratios of FGFR3-S/FGFR-L, TSC2-S/TSC2-L and $R A S G R P 2-b / R A S G R P 2-a$ increased and correlated with augmented invasive and/or proliferative capacity of the AA-derived MDA PCa 2b cells (Supplementary Fig. 6a-c, bottom panels). Collectively, our in vitro studies strongly suggest that the AA-enriched splice variants PIK3CD-S, FGFR3-S, TSC2-S and $R A S G R P 2-b$ promote PCa aggressiveness.

PIK3CD-S isoform promotes activation of AKT/mTOR signalling. As PI3K plays a central role in the PI3K/AKT/mammalian target of rapamycin (mTOR) signalling pathway, we examined the ability of different PI3K $\delta$ isoforms (encoded by PIK3CD- $L$ and $P I K 3 C D-S)$ to activate downstream signalling components within this pathway. SiRNA $\left(\mathrm{siP}_{20}\right)$-mediated knockdown of $P I K 3 K-L$ expression (confirmed by qRT-PCR) led to a drastic decrease in AKT phosphorylation at Thr308 and Ser473 while moderately decreasing (approximately twofold) phosphorylation of mTOR in EA VCaP cells (Fig. 4c, top panel). In contrast, knockdown of PIK3CD-L in AA MDA PCa $2 \mathrm{~b}$ cells (resulting in an increased $P I K 3 C D-S /-L$ ratio confirmed by qRT-PCR) led to a sizable increase (two- to threefold) in phosphorylation of AKT, mTOR and ribosomal protein S6 (S6) (Fig. 4c, top panel).

In parallel experiments, siRNA $\left(\mathrm{siP}_{\mathrm{j}}\right)$-mediated knockdown of PIK3K-S (confirmed by qRT-PCR) in MDA PCa $2 \mathrm{~b}$ cells resulted in a drastic decrease in the phosphorylation status of AKT, mTOR and S6 (Fig. 4c, bottom panel). As expected, treatment of VCaP cells with the siRNA siP ${ }_{j}$ had negligible effects on AKT, mTOR and S6 phosphorylation, as this EA line does not significantly express PIK3CD-S. Taken together, the distinct phosphorylation patterns of AKT, mTOR and S6 in AA and EA PCa cell lines upon selective knockdown of either PIK3CD-L or $P I K 3 C D-S$ again suggested that PIK3CD-S is the more aggressive variant, promoting oncogenic signalling. 

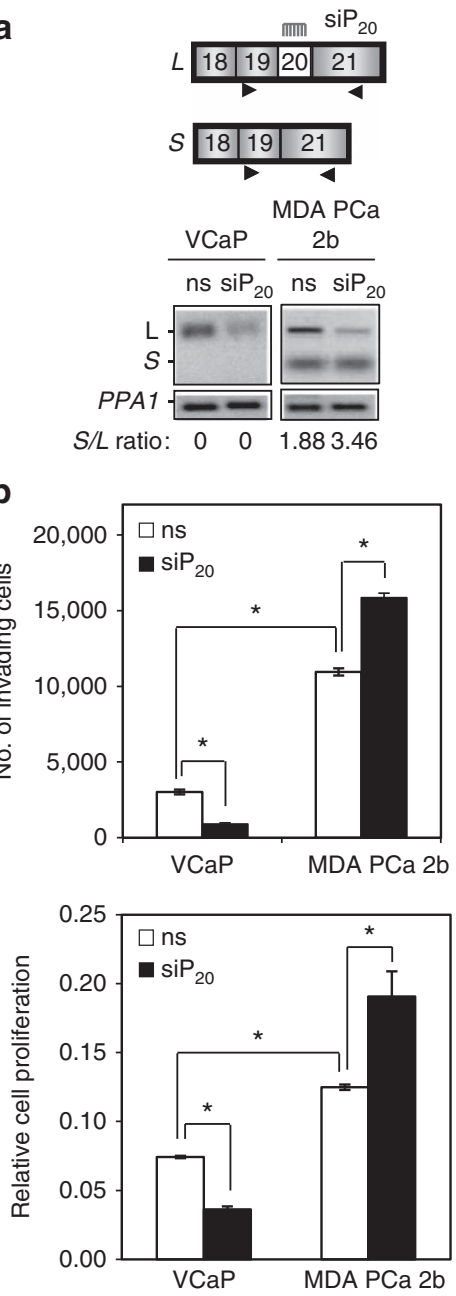

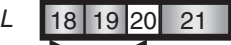

$s$

\begin{tabular}{|l|l|l|}
\hline 18 & 19 & 21 \\
\hline
\end{tabular}

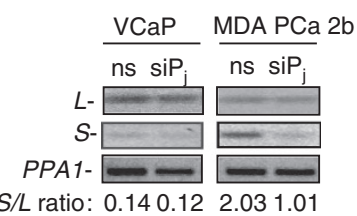

$S / L$ ratio: 0.140 .122 .031 .01
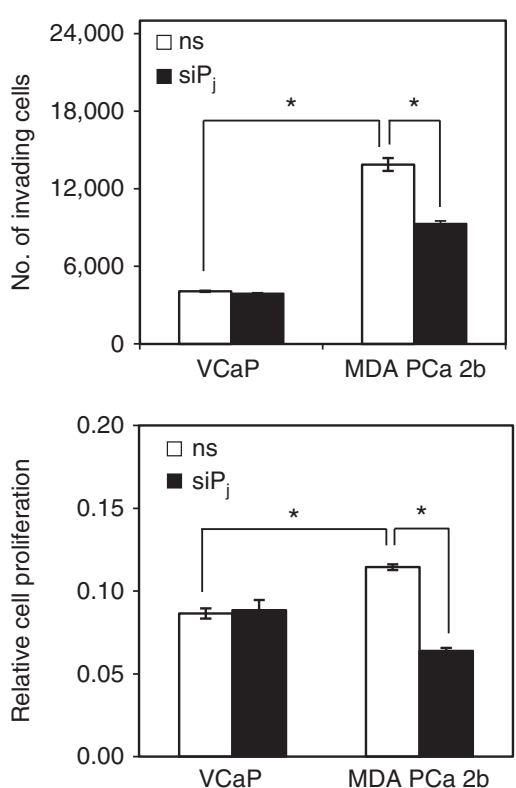

c

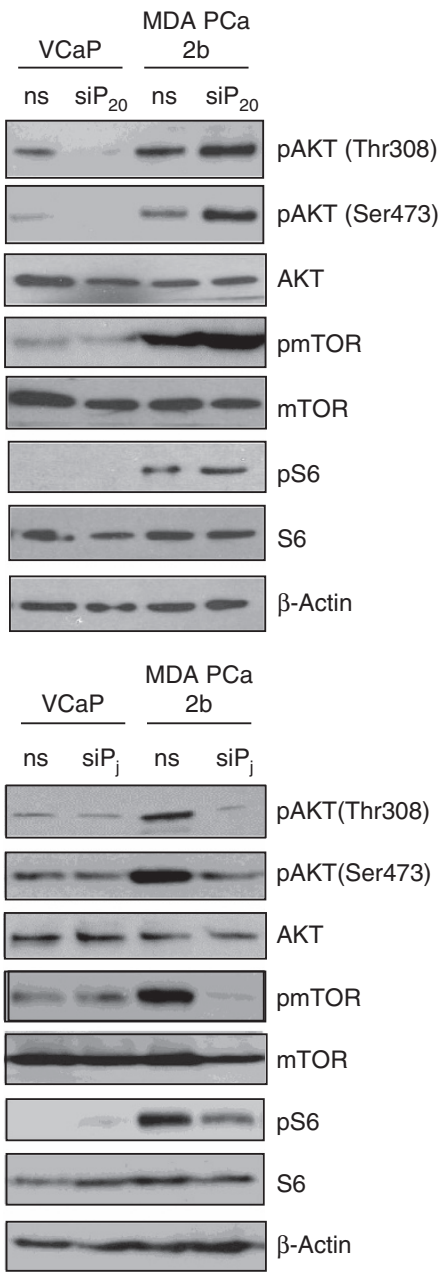

Figure 4 | Selective knockdown of the AA-enriched PIK3CD-S variant or race-independent PIK3CD-L variant has opposing effects on tumorigenesis and AKT/mTOR signalling in AA PCa cells. (a) RT-PCR of PIK3CD-L and PIK3CD-S variants following knockdown in the EA PCa cell line VCaP and AA PCa cell line MDA PCa 2b. Specific knockdown of the PIK3CD-L variant (left panel) was accomplished with an exon 20-targeting siRNA (siP 20 ), while knockdown of the PIK3CD-S variant (right panel) was achieved with an siRNA targeting the region spanning exons 19 and 21 (siP $P_{j}$ ). Closed arrowheads below exons represent primer location for $\mathrm{qRT}-\mathrm{PCR}$ validation of alternatively spliced transcript knockdowns. Knockdown efficiency of siP 20 and siP $\mathrm{P}_{\mathrm{j}} \mathrm{iRNAs}_{\mathrm{N}}$ was determined by the $S / L$ ratio derived from RT-PCR reactions; ns, nonsense siRNA treatment. Representative images of $n=4$ independent knockdown experiments. (b) Proliferation and invasion of VCaP and MDA PCa $2 b$ following knockdown of the PIK3CD-S (left panel) or PIK3CD- $L$ variant (right panel). Data presented as mean \pm s.e.m. from at least three independent experiments for each treatment group. ${ }^{\star} P<0.05$ by ANOVA with post hoc Tukey. Variance was similar among groups being compared. (c) Western blot analysis of AKT/mTOR signalling following knockdown of the PIK3CD-L (top panel) or PIK3CD-S variant (bottom panel) in VCaP and MDA PCa 2b. Level of AKT, mTOR and ribosomal S6 kinase activities is reflected by the amount of phospho-AKT (pAKT), phospho-mTOR (pmTOR) and phospho-ribosomal protein S6 (pS6) immunoblotting, respectively. $\beta$-Actin served as loading control. Representative images from at least three independent western blot experiments.

PI3K $\delta$-S isoform is resistant to SMIs. We tested whether pharmacological inhibition of $\mathrm{PI} 3 \mathrm{~K} \delta$ isoforms represented a potential strategy for ameliorating PCa aggressiveness. CAL-101, an SMI specific for PI3K $\delta$ (refs 37-39), was employed to assess its inhibitory effects on oncogenic signalling and proliferation in EA $\mathrm{VCaP}$ and PC-3 cells that stably overexpressed the His-tagged PI3K $\delta$-S (excluding exon 20) or PI3K $\delta$-L isoform (including exon 20). Equivalent levels of PI3K $\delta$ isoform expression in each cell line was confirmed by western blot with a His tag antibody (Fig. 5a). In both EA cell lines, ectopic overexpression of PI3K $\delta$-S was associated with a two- to threefold greater phosphorylation of AKT and S6 compared with ectopic overexpression of PI3K $\delta$-L (Fig. 5a, absence of CAL-101 treatment). CAL-101 (50 $\mathrm{mg} \mathrm{kg}^{-1}$ ) induced a significant reduction in basal AKT and S6 phosphorylation (Fig. 5a) and a dose-dependent inhibition of proliferation in both EA cell lines overexpressing the PI3K $\delta$-L variant (Fig. 5b).
In contrast, CAL-101 $\left(50 \mathrm{mg} \mathrm{kg}^{-1}\right)$ had negligible effects on inhibiting basal AKT signalling in EA PCa cell lines overexpressing PI3K $\delta$-S, as phosphorylation states of AKT and S6 were comparable to vehicle-treated cells (Fig. 5a). In agreement, 5-bromodeoxyuridine labelling assays demonstrated that proliferation of $\mathrm{VCaP}$ and $\mathrm{PC}-3$ cells ectopically overexpressing $\mathrm{PI} 3 \mathrm{~K} \delta$-S was greater than cells overexpressing PI3K $\delta$-L (Supplementary Fig. 7). Moreover, PI3K $\delta$-S-overexpressing VCaP and PC-3 cells were not effectively inhibited by CAL-101 treatment; only at extreme doses of CAL$101(\geq 30 \mu \mathrm{M})$ was proliferative activity in PC-3 cells significantly impaired (Fig. 5b). In contrast, the AKT inhibitor MK-2206 (ref. 40) dose-dependently decreased proliferation in both PI3K $\delta$-Sand PI3K $\delta$-L-overexpressing $\mathrm{VCaP}$ and PC-3 cells (Fig. 5c). These results suggest that PI3K $\delta$-S-stimulated proliferation is resistant to CAL-101 inhibition in sharp contrast to PI3K $\delta$-L; 
a

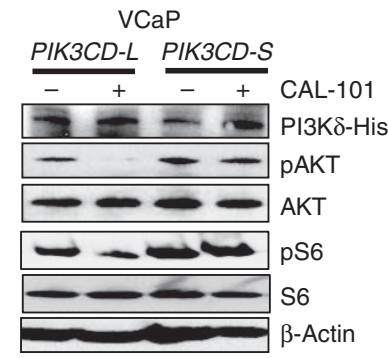

b
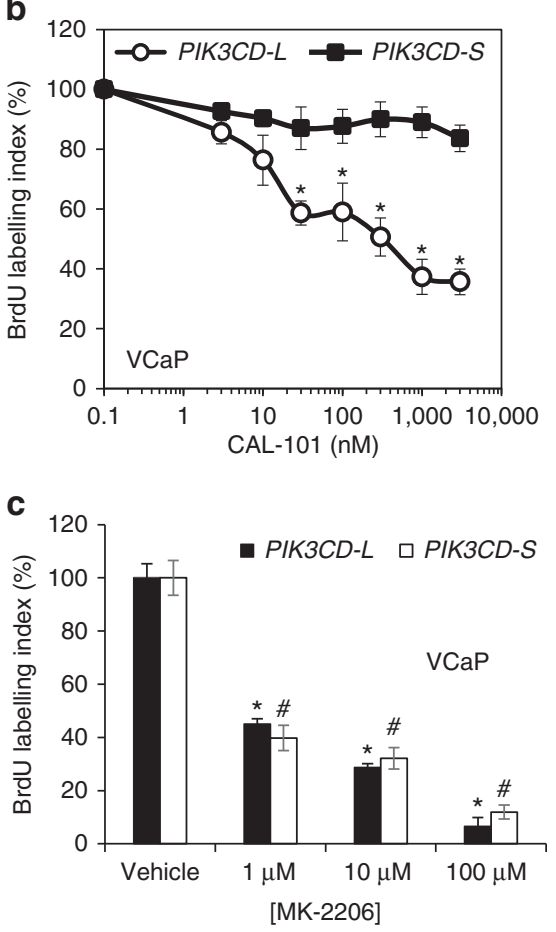

$\mathrm{PC}-3$
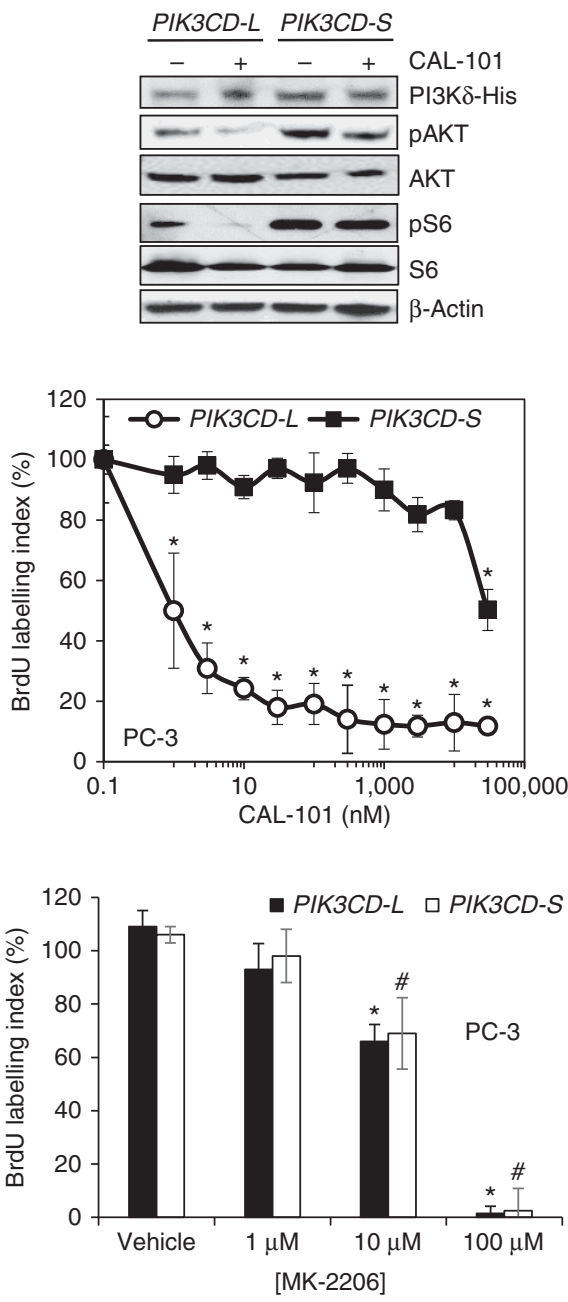

Figure 5 | PI3K $\delta$-S but not PI3K $\delta$-L is resistant to small-molecule inhibition of PI3K/AKT/mTOR signalling and proliferation. (a) Assessment of $\mathrm{PI} K \mathrm{~K} / \mathrm{AKT} / \mathrm{mTOR}$ signalling following treatment with vehicle (saline) or CAL-101 (100 nM, 24 h). PI3K/AKT/mTOR signalling was assessed by western blot analysis with phospho-antibodies to AKT (pAKT) and S6 ribosomal protein (pS6). $\beta$-Actin served as a loading control. His-tag antibody was used to demonstrate equal expression of His-tagged variant PI3K $\delta$ protein in stably transfected cell lines. Representative images from at least three independent western blot experiments. Unprocessed western images shown in Supplementary Fig. 12. (b) Proliferation in VCaP and PC-3 cells stably overexpressing the PIK3CD-S variant or PIK3CD-L variant following treatment with vehicle (saline) or selective PI3K $\delta$ small molecule inhibitor CAL-101 (24 h). Data presented as mean \pm s.e.m. from at least four independent experiments for each treatment group. ${ }^{*}$ Significantly different from $-S$ variant, $P<0.05$ by ANOVA with Dunnett's post hoc test. (c) Treatment of PIK3CD variant-overexpressing cells with vehicle (saline) or selective AKT small molecule inhibitor MK-2206 $(24 \mathrm{~h})$. Proliferation was assessed using a 5-bromodeoxyuridine (BrdU) labelling assay. Data presented as mean \pm s.e.m. from at least four independent experiments for each treatment group. ${ }^{*}$ Or ${ }^{\#}$ significantly different from corresponding vehicle control, $P<0.05$ by ANOVA with Dunnett's post hoc test. Variance was similar among groups being compared.

while inhibition of AKT, which is downstream of $\mathrm{PI} 3 \mathrm{~K} \delta$-S, effectively blocked proliferation.

To examine the effects of $P I K 3 C D$ splice variants on tumour growth in vivo, we subcutaneously injected $2 \times 10^{6} \mathrm{PC}-3$ cells stably overexpressing equivalent amounts of the PI $3 \mathrm{~K} \delta$-L or PI3K $\delta$-S (missing exon 20) isoform into the left hind flank of nonobese diabetic-severe combined immunodeficient (NOD-SCID) mice. Mice harbouring $\mathrm{PI} 3 \mathrm{~K} \delta$-L-overexpressing or PI3K $\delta$-S-overexpressing PC-3 cell xenografts were administrated either vehicle (phosphate-buffered saline) or CAL-101 $\left(50 \mathrm{mg} \mathrm{kg}^{-1}\right)$ by daily intraperitoneal (i.p.) injection. CAL-101 treatment for 30 days significantly reduced the growth of $\mathrm{PI} 3 \mathrm{~K} \delta$-L-expressing xenografts compared with the vehicle treatment (Fig. 6a,b). In contrast, mice with xenografts of PI3K $\delta$-Sexpressing cells had negligible suppression of their xenograft growth following CAL-101 treatment compared with vehicletreated animals (Fig. 6a,b).

We further examined the inhibitory effects of CAL-101 on $\mathrm{PI} 3 \mathrm{~K} \delta$ isoforms in an in vivo tumour metastasis model. The $1 \times 10^{6}$ PIK3CD-L- or PIK3CD-S-overexpressing PC-3 cells were injected into the tail vein of NOD-SCID mice, and animals were subsequently administrated with vehicle or CAL-101 (50 $\mathrm{mg} \mathrm{kg}^{-1}$ ) via i.p. injection (3 times per week). After 8 weeks, vehicle-treated mice carrying PI $3 \mathrm{~K} \delta$-L-overexpressing cells developed prominent tumour metastases in the lungs (Fig. $6 \mathrm{c}, \mathrm{d}$ ), while the CAL-101 treatment group exhibited a $>50 \%$ reduction $(P<0.05)$ of metastases (Fig. 6c,d). In comparison, CAL-101 treatment failed to significantly inhibit tumour metastases in mice harbouring PI3K $\delta$-S-overexpressing cells (Fig. 6c,d). Noteworthy, the size of lung metastases (average area of nodules) in mice 
a

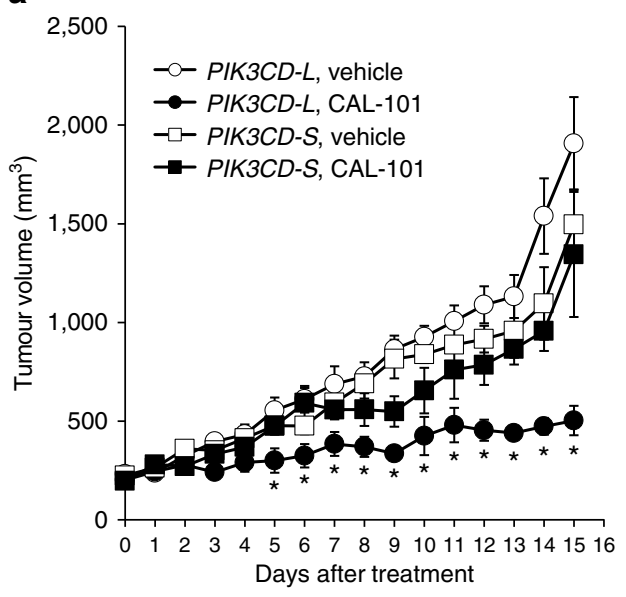

C

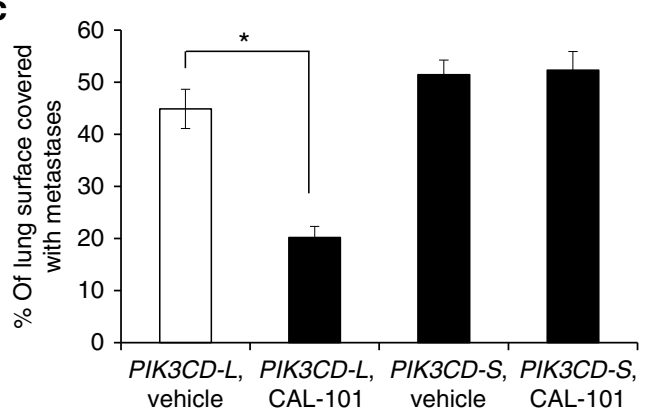

b

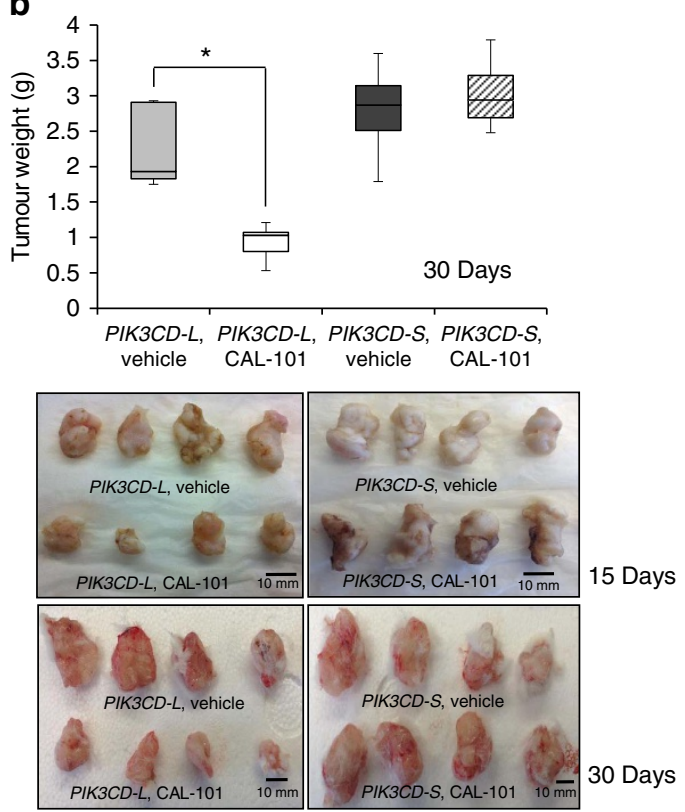

d

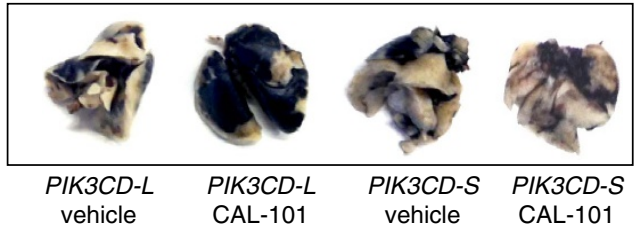

Figure 6 | PI3K $\delta$-S but not PI3K $\delta$-L is resistant to small-molecule inhibition of xenograft growth and metastasis. (a) Growth of PC-3 cells stably overexpressing the PIK3CD-S variant in NOD-SCID mice is resistant to CAL-101 treatment $\left(50 \mathrm{mg} \mathrm{kg}^{-1}\right.$ i.p. 3 times a week). In contrast, growth of PC-3 cells stably overexpressing the PIK3CD-L variant is sensitive to CAL-101 treatment. Data represent the mean tumour size \pm s.e.m. of $n=10$ independent mice for each treatment group at each time point. *Significantly different from saline-treated group, $P<0.05$ by ANOVA with post hoc Tukey. Variance was similar among groups being compared. (b) Tumour weights and gross morphology of tumour xenografts from a. Box-and-whisker plot represents mean xenograft weight in mice after 30-day vehicle or CAL-101 treatment. ${ }^{\star} P<0.05$ by ANOVA with Dunnett's post hoc test; $n=10$ independent mice for each treatment group. Variance was similar among groups being compared. (c) Quantification of lung metastases in NOD-SCID mice. PC-3 cells stably overexpressing PIK3CD-L or PIK3CD-S were injected into the tail vein of NOD-SCID mice treated with vehicle or CAL-101 (50 mg kg ${ }^{-1}$ i.p. 3 times a week). After 8 weeks, lungs were collected and stained with India ink and bleached with Fekete's solution for visualization of metastatic nodules (white-coloured areas). Data presented as mean \pm s.e.m. of $n=10$ for each treatment group. ${ }^{\star} P<0.05$ by ANOVA with Dunnett's post hoc test. Variance was similar among groups being compared. (d) Representative India ink-stained lungs from treatment groups analysed in c.

harbouring $\mathrm{PI} 3 \mathrm{~K} \delta$-S-overexpressing cells was slightly greater $(\sim 15 \%)$ compared with animals with $\mathrm{PI} 3 \mathrm{~K} \delta$-L-overexpressing cells, although not statistically significant $(P>0.05)$. Taken together, the in vitro and in vivo functional studies suggest that SMIs such as CAL-101 (competitive ATP binding inhibitors ${ }^{39,41}$ ) may be ineffective against the PI $3 K \delta-S$ isoform in AA PCa.

Cell-free PI3K $\boldsymbol{\delta}$ isoform kinase assay. The consequence of excluding exon 20 (168 bp) in the PIK3CD-S variant is an in-frame deletion of 56 amino acids (residues 810-865) in the catalytic domain of the PI3K $\delta$-S isoform (Fig. 7a). To gain further insight into the functional differences between PI3K $\delta$ isoforms, the interaction of PI $3 \mathrm{~K} \delta-\mathrm{L}$ and $-\mathrm{S}$ with regulatory subunit $\mathrm{p} 85 \alpha$ was investigated. Whole cell lysates from transfected PC-3 cells overexpressing $\mathrm{p} 85 \alpha$ and either His-tagged $\mathrm{PI} 3 \mathrm{~K} \delta$-S or PI3K $\delta$-L were subjected to western analysis, demonstrating that each cell line expressed equivalent levels of their respective PI3K $\delta$ isoform as well as equal p85 $\alpha$ expression (Fig. $7 \mathrm{~b}$, left panel). Interestingly, co-immunoprecipitation (co-IP) of the $\mathrm{PI} 3 \mathrm{~K} \delta / \mathrm{p} 85 \alpha$ complex from whole cell lysates using an anti-His antibody demonstrated that $\mathrm{p} 85 \alpha$ bound with three- to fourfold greater proficiency to
PI3K $\delta$-L compared with $\mathrm{p} 85 \alpha$ binding to $\mathrm{PI} 3 \mathrm{~K} \delta$-S (Fig. $7 \mathrm{~b}$, right panel, column E). Binding proficiency was inversely correlated with $\mathrm{PI} 3 \mathrm{~K} \delta$ isoform kinase activity (Fig. 7c, right panel).

Next, PI3K $\delta$ isoforms were purified from the lysates of PC-3 cells overexpressing either His-tagged PI $3 \mathrm{~K} \delta$-S or PI3K $\delta$-L using Ni-NTA resin columns. As shown in Fig. $7 \mathrm{~d}$ (left and middle panels), $\mathrm{PI} 3 \mathrm{~K} \delta$-S and $-\mathrm{L}$ purification was verified by western blotting using anti-His or anti-PI3K $\delta$ antibody. Moreover, the $\mathrm{Ni}-\mathrm{NTA}$ resin column approach resulted in the isolation of $\mathrm{PI} 3 \mathrm{~K} \delta$ isoforms that were no longer bound to p85 $\alpha$ (Fig. 7d; far right panel, column E). Early reports have demonstrated that $\mathrm{PI} 3 \mathrm{~K} \alpha / \mathrm{p} 85$ and $\mathrm{PI} 3 \mathrm{~K} \beta / \mathrm{p} 85$ complexes are obligate and extremely stable, being able to withstand high concentrations of urea or detergent $^{42-44}$. Our finding that $\mathrm{PI} 3 \mathrm{~K} \delta$ and $\mathrm{p} 85 \alpha$ co-exist as monomers and complexes was unexpected. It should be noted, however, that the nature of interaction between PI3K $\delta$ and $\mathrm{p} 85$ is less well established, as these two proteins have been shown in separate studies to either form a stable obligate complex ${ }^{42}$ or coexist complexed together and uncomplexed from each other ${ }^{45}$.

Purified PI3K $\delta$ isoforms (minus $\mathrm{p} 85 \alpha$ ) were incubated with vehicle, nonselective PI3K inhibitor wortmannin $(100 \mathrm{nM})^{46}$ or $\mathrm{PI} 3 \mathrm{~K} \delta$-specific inhibitor CAL-101 $(100 \mathrm{nM})$, and subjected to a 


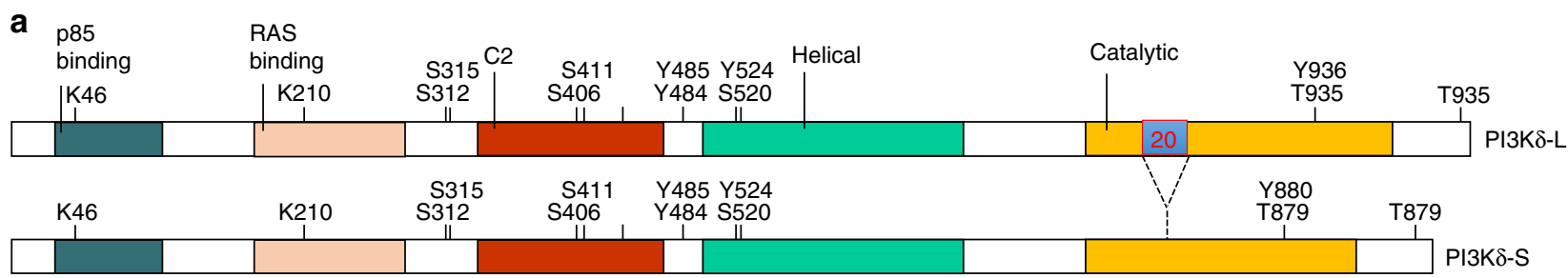

b

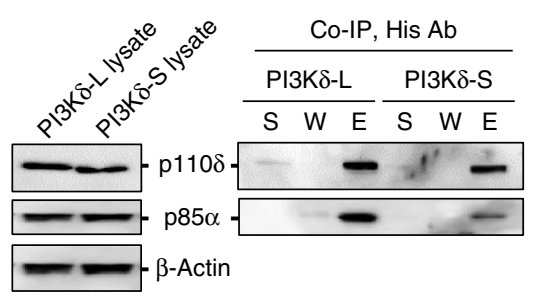

C

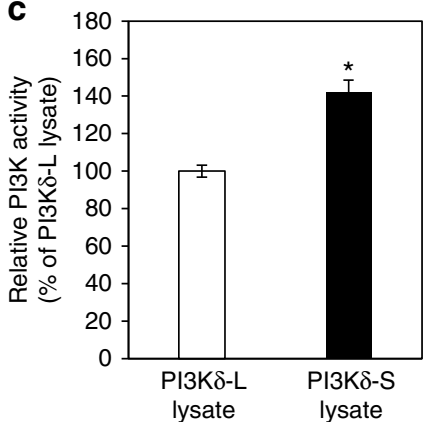

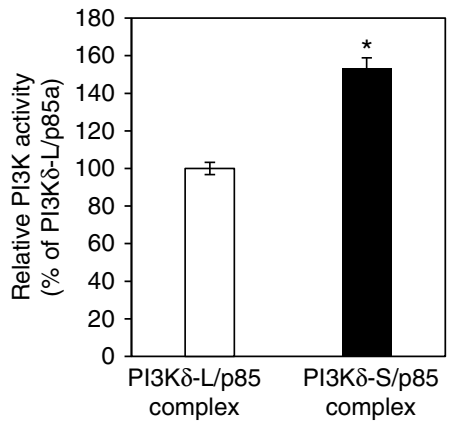

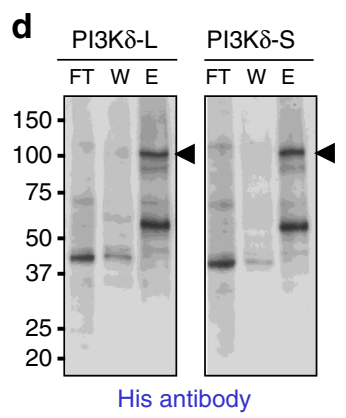

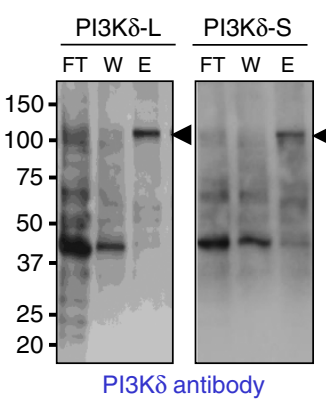

$\mathrm{PI} 3 \mathrm{~K} \delta$ antibody

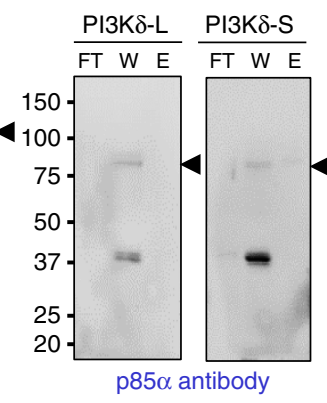

e

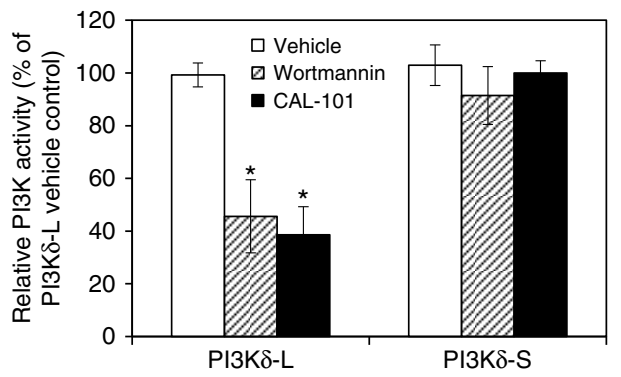

Figure 7 | Cell-free kinase assay of PI3K $\delta$ isoforms and small-molecule inhibition. (a) Schematic representation of protein domains of PI3K $\delta$-L and $-S$ isoforms. Adaptor (p85)-binding, RAS binding, C2, helix and catalytic domains are highlighted. Phosphorylation and ubiquitination sites ( $\mathrm{S}, \mathrm{T}$, Y and K) and the region encoded by exon 20 (56 amino acids) residing in the catalytic domain are indicated. (b) Co-immunoprecipitation (Co-IP) of His-tagged PI3K $\delta / p 85 \alpha$ complex from transfected PC-3 cells followed by western blot, and (c) PI3K activity assays. S, supernatant; W, wash fraction; E, eluted fraction. Anti-His antibody was used in the Co-IP experiments, and anti-His, anti-p85 $\alpha$ and anti-actin antibodies were used in the western blotting. *Significantly different kinase activities in total lysates of PI3K $\delta$-S versus PI3K $\delta$-L-expressing cells, or purified $\mathrm{PI} 3 \mathrm{~K} \delta$-S $/ \mathrm{p} 85 \alpha$ versus $\mathrm{PI} 3 \mathrm{~K} \delta$ - L/p85 $\alpha$ complexes. $P<0.05$ using two-sided Student's $t$-test. Data presented as mean \pm s.e.m. of $n=4$ for each treatment group. (d) Purification of His-tagged PI3K $\delta$-L and $-\mathrm{S}$ isoforms. Western blot analysis of Ni-NTA resin-purified PI3K $\delta$ isoforms from transfected PC-3 cells using His and PI3K $\delta$ antibodies. FT, flow-through; W, wash fraction; $\mathrm{E}$, eluted fraction. Closed arrowheads indicate PI3K $\delta$ isoforms. (e) Cell-free kinase assay of $L$ and $S$ isoforms of PI3K $\delta$ in the presence of vehicle (phosphate-buffered saline (PBS)), $100 \mathrm{nM}$ wortmannin or $100 \mathrm{nM} \mathrm{CAL-101.}{ }^{\star}$ Significantly different from vehicle control-treated PI3K $\delta$-L isoform. $P<0.05$ by ANOVA with Dunnett's post hoc test. Data presented as mean \pm s.e.m. from at least four independent experiments for each treatment group. Blots in $\mathbf{b}, \mathbf{d}$ are representative from at least three independent experiments with similar results. Variance was similar among groups being compared. Unprocessed western images are shown in Supplementary Fig. 13.

PI3K activity assay. In the absence of bound $\mathrm{p} 85 \alpha$, kinase activity of $\mathrm{PI} 3 \mathrm{~K} \delta$ - $\mathrm{L}$ was equivalent to $\mathrm{PI} 3 \mathrm{~K} \delta$-S (Fig. $7 \mathrm{e}$, compare vehicle treatments). In agreement, siRNA-mediated knockdown of p85 $\alpha$ in wild-type VCaP and PC-3 cells was associated with an increase in invasive activity (Supplementary Fig. 8). Remarkably, wortmannin and CAL-101 significantly inhibited the activity of the $\mathrm{PI} 3 \mathrm{~K} \delta$-L isoform, but not the PI $3 \mathrm{~K} \delta$-S isoform (Fig. $7 \mathrm{e}$ ). These results demonstrate that $\mathrm{PI} 3 \mathrm{~K} \delta$-S maintains kinase activity even in the presence of SMIs, supporting the in vitro and in vivo results (Figs 5 and 6).

\section{Discussion}

The phenomenon of DS, much less global DS events, has not been adequately explored as a possible mechanism underlying $\mathrm{PCa}$ disparities. Potential involvement of the constitutively active $A R$ $V 7$ splice variant in PCa disparities has been suggested in a recent study. Selective downregulation of miR-212 observed in AA PCa is correlated with upregulation of splicing factor hnRNP-H1, upregulation of $A R-V 7$ and antiandrogen resistance in PCa cell lines ${ }^{47}$. In contrast to this localized splicing event, our study reveals that DS on a global scale may be a critical molecular mechanism underlying PCa disparities. In a comparison of AA PCa versus EA PCa, DS events were found to be highly prevalent in cancer-associated genes and pathways (Supplementary Data 1 and 2). Interestingly, the number of genes harbouring predicted DS events $(2,520$ genes) was $\sim 3 \times$ greater than the number of differentially expressed, but not differentially spliced, genes (886 genes). These findings have two major implications. First, alternative/aberrant splicing of pre-mRNAs may have a greater role than differential gene expression in driving PCa disparities. Second, predicted DS events identified in our study were statistically overrepresented in oncogenic signalling pathways. In many cases, these same pathways are known to harbour a 
preponderance of gene mutations across different cancer types ${ }^{27-29}$. Hence, DS adds another layer of complexity to the existing molecular repertoire (gene mutation, expression, methylation ${ }^{7}$ ) driving AA PCa aggressiveness.

Studies on AS indicate that approximately half of such events occurring in the coding sequence are in-frame, while the remaining events are frameshifts leading to truncated or extended C-terminal proteins ${ }^{48,49}$. Remarkably, $70 \%$ of AA-enriched/specific variants in our composite oncogenic signalling pathway (Fig. 2) were in-frame, including PIK3CD-S, FGFR2-S, FGFR3-S, TSC2-S, RASGRP2-b, ATM-S and GSK3-S (Supplementary Table 2). In comparison, only $27.3 \%$ of EA-enriched/-specific DS events in our composite oncogenic signalling pathway exhibited in-frame preservation (Supplementary Table 2), while the remaining EA-enriched DS variants, including ITGA4-S, MET-S, NF1-S, RASGRP2- $a$, mTOR-S and BAK1-S, were frameshifted. Why the vast majority of DS events appear to be in-frame in AA PCa versus frame-shifted in EA PCa remains unresolved (Supplementary Table 1). Presumably, the preponderance of AA in-frame events detected in oncogenic signalling pathways may be contributing to the more aggressive nature of AA PCa. Possible mechanisms that could drive differences in AS events include differential expression of trans-acting splicing factors ${ }^{47}$ and/or single-nucleotide polymorphisms in cis-acting splicing elements of alternatively spliced genes ${ }^{50}$. In fact, a number of splicing factor mRNAs appear to be overexpressed (SRSF2, SRSF7) in AA $\mathrm{PCa}$ compared with EA PCa ${ }^{9,10}$. Regarding the in-frame variants (PIK3CD-L, FGFR3-L and TSC2-L) detected in EA PCa, each conferred a less aggressive oncogenic phenotype compared with the corresponding in-frame variants detected in AA PCa (PIK3CD-S, FGFR3-S and TSC2-S).

Approximately one-third of the AA-enriched/-specific variants identified in AA PCa were likewise present in patient-matched NP specimens, whereas the remaining AA-enriched/-specific variants found in $\mathrm{PCa}$ were absent in patient-matched NP specimens and thus appear to be de novo events (occurring as NP evolved into $\mathrm{PCa}$ ). Accordingly, the AA-enriched/-specific variants already present in NP specimens have the potential to serve as inherent 'at-risk alleles' for poor PCa prognosis in AAs. In comparison, the de novo appearance of tumour-specific variants may drive poorer outcomes. PIK3CD-S would be an example of a potential AA 'at-risk allele' contributing to increased $\mathrm{PCa}$ aggressiveness upon disease presentation. Indeed, ectopic overexpression of the AA-enriched PIK3CD-S in PCa cell lines was demonstrated to enhance oncogenic potential (increased invasion, proliferation and $\mathrm{AKT} / \mathrm{mTOR}$ signalling) compared with the corresponding EA-enriched PIK3CD-L. Moreover, genetic manipulation of AA MDA PCa $2 \mathrm{~b}$ cells to favour expression of the $-S$ variant over the $-L$ variant likewise increased oncogenic behaviour. Conversely, genetic manipulation in the opposite direction decreased oncogenic behaviour. Interestingly, survival plots generated from The Cancer Genome Atlas (TCGA) RNA-sequencing data demonstrate that a high $S / L$ ratio is associated with significantly worse survival for PCa and trending for worse survival in both breast and colon cancer (Supplementary Fig. 9). Survival plots were not stratified by race as this information is not currently available in TCGA. Given the number of patients analysed, it seems highly probable that high $S$ / $L$ ratio values may also be associated with a subset of EA patients, suggesting that PIK3CD-S may be useful in predicting survival in all patients irrespective of race. Besides PIK3CD-S, an additional 732 potential 'at-risk alleles' (for example, ITGA4-L, MET-L) were identified that may be associated with poor PCa prognosis in AAs. Further experimentation is needed to investigate whether these variants can serve as novel biomarkers to address $\mathrm{PCa}$ disparities. In contrast to the 'at-risk alleles', AA-enriched variants FGFR-S and TSC2-S were detected in AA PCa, but not in patient-matched NP specimens. The appearance of these de novo variants during PCa formation may contribute to driving the more aggressive PCa phenotype observed in the AA population, since in vitro genetic manipulation favoring expression of these $-S$ variants over the $-L$ variants promoted oncogenesis in MDA PCa $2 \mathrm{~b}$ cells. It is noteworthy that three PCa-associated splice variants identified in previous studies, $B c l-x L, F G F R 2-I I I c$ and TMPRSS2-ERG +72 (refs 14,15,20), did not exhibit DS in our AA PCa versus EA PCa comparison (Supplementary Data 1), suggesting that these variants may contribute to $\mathrm{PCa}$ progression/aggressiveness in a race-independent manner.

The identification of PIK3CD-S, a variant newly discovered and cloned in our study, as an 'at-risk allele' for PCa aggressiveness is germane given that PI3K signalling is aberrantly activated in a variety of cancers and PI3K inhibitors have been developed as targeted therapeutics ${ }^{51,52}$. Class IA PI3Ks consist of three isoforms, including $\mathrm{PI} 3 \mathrm{~K} \alpha, \mathrm{PI} 3 \mathrm{~K} \beta$ and $\mathrm{PI} 3 \mathrm{~K} \delta$. Unlike ubiquitously expressed PI3K $\alpha$ and PI3K $\beta, \mathrm{PI} 3 \mathrm{~K} \delta$ appears to be preferentially expressed in leukocytes ${ }^{53,54}$. Previous studies have revealed a crucial role of $\mathrm{PI} 3 \mathrm{~K} \delta$ in lymphoid and myeloid malignancies 39,55 . Interestingly, accumulating evidence suggests a functional role of $\mathrm{PI} 3 \mathrm{~K} \delta$ in promoting nonhaematologic tumours as well. For example, overexpression of PIK3CD mRNA and/or $\mathrm{PI} 3 \mathrm{~K} \delta$ protein has been detected in glioblastoma ${ }^{32}$, neuroblastoma ${ }^{30}$, breast cancer ${ }^{33}$ and $\mathrm{PCa}^{31}$, and PIK3CD overexpression has been implicated in promoting cell growth/ survival in breast cancer and neuroblastoma ${ }^{30,33}$. Consistent with these findings, our immunohistochemistry experiments using a pan-PI3K $\delta$ antibody likewise revealed strong expression of PI3K $\delta$ protein in PCa specimens as well as PCa, breast cancer and colon cancer cell lines (Supplementary Fig. 10). Importantly, our study provides greater granularity by being the first to demonstrate the relationship between expression of a race-enriched PIK3CD splice variant and cancer aggressiveness as well as resistance to SMIs targeting $\mathrm{PI} 3 \mathrm{~K} \delta$.

Aberrant pre-mRNA splicing has recently been demonstrated to mediate therapeutic resistance in multiple cancer types. For example, the constitutively active $A R-V 7$ variant (lacking exonic sequences encoding the ligand binding domain) confers resistance to enzalutamide and abiraterone acetate in castrationresistant $\mathrm{PCa}$ patients $^{56}$. In addition, melanoma patients harbouring $B R A F$ splice variants encoding protein isoforms that are missing the RAS-binding domain exhibited resistance to the RAF inhibitor vemurafenib ${ }^{57}$. Noteworthy, these studies did not investigate whether variant expression and therapeutic responsiveness stratified along racial lines. We now provide evidence that AA-enriched PIK3CD-S imparts PCa cell lines with significant resistance to SMIs targeting PI $3 \mathrm{~K} \delta$, as demonstrated in both in vitro assays and preclinical mouse models of PCa. This short variant is missing exon 20, encoding a 56-amino acid segment that is present in PIK3CD-L. Amino acids residing in the exon 20-encoded cassette appear critical for the docking of CAL-101 and wortmannin. Indeed, molecular modelling studies predict that Glu826 and Val828 (missing in PI3K $\delta$-S) undergo hydrogen bonding with CAL-101 (ref. 58). Noteworthy, overall response of indolent lymphoma and chronic lymphocytic leukaemia to CAL-101 ranges from 48 to $81 \%$ (refs 59-61). Given our findings, it would be of interest to determine whether patients with primary resistance harbour malignant cells expressing CAL-101-resistant $\mathrm{PI} 3 \mathrm{~K} \delta$-S, while responsive patients harbour malignant cells expressing CAL-101-sensitive $\mathrm{PI} 3 \mathrm{~K} \delta$-L.

P85 regulatory subunits are known binding partners of class I PI3Ks, resulting in protein stabilization and suppression of basal kinase activity ${ }^{62,63}$. Somatic mutations in PIK3R1 (encoding 
$\mathrm{p} 85 \alpha)$ have been identified that abrogate the inhibitory action of p $85 \alpha$ on PI3K $\alpha$ in cancers ${ }^{64,65}$. Our cell-free assays demonstrated that $\mathrm{p} 85 \alpha$ binds more efficiently with PI $3 \mathrm{~K} \delta$-L compared with PI3K $\delta$-S. This interaction appears to be responsible for the lower kinase activity exhibited by PI $3 \mathrm{~K} \delta$-L, as disruption of binding led to a long isoform with increased kinase activity comparable to PI3K $\delta$-S (Fig. 7c,e). The amino acid Asn334 located on the $\mathrm{N}$-terminal side of PI3K $\delta$ has been postulated to serve as a critical contact point with $\mathrm{p} 85 \alpha$ (ref. 66). Our findings suggest that amino acids $810-865$, encoded by exon 20 and missing in PI $3 K \delta$-S, may also contain essential amino acids required for efficient coupling to $\mathrm{p} 85 \alpha$. Alternatively, amino acids $810-865$ permits $\mathrm{PI} 3 \mathrm{~K} \delta$-L to adopt a conformation where Asn334 (and other amino acids) is available to interact with $\mathrm{p} 85 \alpha$.

The identification and functional validation of global AS in cancer pathogenesis remains challenging and largely unexplored. We have undertaken such an analysis in the context of race-related aggressive $\mathrm{PCa}$ and identified a large number of DS events in cancer-associated pathways in EA and AA PCa, with a subset of these events also being detected in patient-matched NP specimens. These events will have both biological and clinical consequences, case in point $P I K 3 C D-S$. The identification of novel splice variants as biomarkers and/or development of therapeutics targeting protein isoforms have the potential to reduce cancer disparities.

\section{Methods}

Materials. EA PCa cell lines LNCaP (CRL-1740), VCaP (CRL-2876) and PC-3 (CRL-1435), and AA PCa cell line MDA PCa 2b (CRL-2422) were obtained from the American Type Tissue Collection (ATCC, Manassas, VA, USA), authenticated at the ATCC by short tandem repeat profiling of multiple unique genetic loci, and tested negative for mycoplasma. Primer sequences for RT-PCR are provided in Supplementary Table 3. The siRNAs were purchased from GE Dharmacon (Lafayette, CO, USA) and sequences are as follows: nonsense, $5^{\prime}$-CCA AAUUAU ACCUACAUUGCU-3'; $\operatorname{siP}_{20}, 5^{\prime}$-CCAACAUCCAACUCAACAA- $3^{\prime} ; \mathrm{siP}_{\mathrm{j}}$,

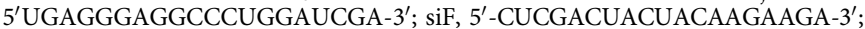
siTSC2-ex20, CUGCGCUAUAAAGUGCUCA-3'; siRASGRP2-ex11, 5'-CCACAU CUCACAGGAAGAA- $3^{\prime}$. siPIK3R1 Smart Pool (5'-AGUAAAGCAUUGUGUC AUA-3', 5' -CCAACAACGGUAUGAAUAA- $3^{\prime}, 5^{\prime}$-GACGAGAGACCAAUAC UUG-3', $5^{\prime}$-UAUUGAAGCUGUAGGGAA A-3').

Collection of PCa clinical specimens. Prostate biopsy samples were collected at the George Washington University Medical Faculty Associates according to an institutional review board-approved protocol (IRB no. 020867). Informed consent was obtained from all study participants. High-quality PCa and patient-matched normal prostate (NP) biopsy cores from each of 20 AA and 15 EA primary PCa patients were collected and processed for the exon array analysis. PCa cores were determined by a pathologist to have Gleason scores of 6-7 (17 AA and $13 \mathrm{EA}$ ) or 8-9 (3 AA and 2 EA), while NP cores were diagnosed negative for cancer. There were no significant differences $(t$-test, $P>0.05)$ between the two racial groups with respect to age (average age for AAs was $62.3 \pm 8.2$, average age for EAs was $63.3 \pm 9.2$ ) and Gleason score (range 6-8; Fisher's exact test, $P>0.05$ ). No distant metastasis was detected in the enrolled patients.

Exon array and statistical analyses. Total RNA was purified from PCa and patient-matched NP biopsy cores using the RNeasy micro kit as per manufacturer's protocol (Qiagen, Valencia, CA, USA). Briefly, total RNA samples were extracted using Trizol reagent, then treated with DNase I and further purified by RNeasy MinElute spin column. High-quality RNA isolation was confirmed by using the Agilent Bioanalyzer as per the manufacturer's protocol (Agilent Technologies, Santa Clara, CA, USA). For exon array analysis, $1 \mu \mathrm{g}$ of purified RNA sample from each biopsy core was interrogated with the Affymetrix Human Exon 1.0 ST GeneChip (Santa Clara, CA, USA). Exon microarray data can be assessed at GEO (Gene Expression Omnibus) using accession number GSE64331. The exon array raw data were subjected to quantile normalization, GC-content adjustment, RMA background correction and $\log _{2}$ transformation using Partek Genomics Suite 6.6 software (Partek Incorporated, St Louis, MO, USA). Detection of differential expression at the gene level (gene-wise analysis) was performed in Partek using the One-Step Tukey's Biweight algorithm for detection of outlier probe-sets. Statistical analysis of exon expression data was based on ANOVA with multiple-correction testing using $10 \%$ false discovery rate (FDR) ${ }^{67}$ criterion. DS events were modelled using the AS ANOVA algorithm ${ }^{26}$ implemented in Partek together with selection of probe-sets exhibiting significant AS score determined at a $2 \%$ FDR. Principal component analysis plots and two-dimensional hierarchical clustering of exon-level data were performed using Partek. DS events were tested for statistical overrepresentation in canonical signalling pathways by Fisher's exact test using the Ingenuity Pathway Analysis (IPA) program (Ingenuity Systems, Redwood City, CA, USA).

RT-PCR validation of AS variants in AA and EA PCa. QRT-PCR was performed using the 7300 Real-Time PCR System (Applied Biosystems, Foster City, CA, USA) to validate and quantify AS events. Primers were designed to amplify the flanking regions of skipped exons or the junctions across catenated exons of variant mRNAs (Fig. 3b). Amplified RT-PCR products were quantified and normalized to housekeeping genes, EIF1AX and PPA1, using the $\triangle \Delta \mathrm{Ct}$ approach ${ }^{9,10}$. Primer sequences for RT-PCR validation are listed in Supplementary Table 3.

Molecular cloning of PIK3CD-S and PIK3CD-L variants. RT-PCR was performed to amplify $P I K 3 C D-L$ and $P I K 3 C D-S$ transcript variants from purified RNA of PC-3, VCaP and MDA PCa 2b cells (ATCC, Manassas, VA). PC-3 and VCaP cells were maintained in Dulbecco's modified Eagle's medium (DMEM; Life Technologies, Gaithersburg, MD, USA) supplemented with $10 \%$ fetal bovine serum (FBS), while MDA PCa 2b cells were grown in BRFF-HPC-1 medium (AthenES, Baltimore, MD, USA) supplemented with $20 \%$ FBS. All the cell lines were grown at $37^{\circ} \mathrm{C}$ and $5 \% \mathrm{CO}_{2}$. Primers were designed according to the National Center for Biotechnology Information (NCBI) reference sequences of PIK3CD mRNA (NM_005026.3). The forward primer contained the start codon (bold) ( $5^{\prime}$-ATGCCCCCTGGGGTGGACT- $3^{\prime}$ ) and the reverse primer was upstream of the stop codon $\left(5^{\prime}\right.$-CTGCCTGTTGTCTTTGGACA-3 $\left.3^{\prime}\right)$. Full-length PCR products were ligated into pcDNA3.1/V5-His TOPO vector (K4800-01, Invitrogen, Grand Island, NY, USA) using the manufacturer's protocol. A total of 8-10 independent clones were selected for each of the amplified PIK3CD-L and PIK3CD-S variants and sequence verified. The consensus sequences of PIK3CD-S and PIK3CD-L mRNAs were deposited to GeneBank (accession number KU612116 and KU612117). The plasmids pcDNA3.1-PIK3CD-L/V5-His and pcDNA3.1PIK3CD-S/V5-His were individually transfected into the PCa cell lines (VCaP and PC-3) using the cationic lipid-mediated method $^{9}$ to establish stable cell lines overexpressing PIK3CD-L or PIK3CD-S.

SiRNA-mediated knockdown in PCa cell lines. VCaP and MDA PCa 2b cells were grown in DMEM with $10 \%$ FBS for $24 \mathrm{~h}$, and then were transfected for $24 \mathrm{~h}$ with siRNAs (50 nM) designed to target splice variants of PIK3CD, FGFR3, TSC2 or RASGRP2 using DharmaFECT4 transfection reagent (Dharmacon), according to the manufacturer's protocol. The in vitro functional assays, including cell proliferation and invasion, were performed following siRNA transfections for $24 \mathrm{~h}$. Cell proliferation and invasion assays were performed using 5-bromodeoxyuridine Cell Proliferation Assay kit (Calbiochem, Billerica, MA, USA) and the Matrigel Invasion Chambers (BD Biosciences, San Jose, CA, USA), respectively, as per the manufacturers' protocol ${ }^{9,10}$.

Antibodies. Antibodies used in western blot analysis were rabbit monoclonal antibodies for pAKT ${ }^{\mathrm{Tyr} 308}, \mathrm{pAKT}{ }^{\text {Ser473 }}$, AKT, pmTOR, mTOR, pS6 and S6 (2965, 4058, 4691, 2971, 2983, 4857 and 2983, Cell Signaling Technology, Danvers, MA, USA), rabbit polyclonal antibodies for His-tag (ab18184, Abcam, Cambridge, MA, USA), HA-tag, PI3K $\delta$, p $85 \alpha$ and $\beta$-actin (sc-7392, sc-55589, sc-1637 and sc-4778, Santa Cruz Biotechnology, Santa Cruz, CA, USA). Horseradish peroxidase-conjugated secondary antibodies for rabbit and mouse IgG were purchased from Southern Biotech (Birmingham, AL, USA).

In vivo xenograft and metastasis models. All animal work was approved by the George Washington University institutional animal care and use committee (protocol A272). Male NOD-SCID mice, 4-6 weeks old, were purchased from the Jackson Laboratory (Bar Harbor, ME, USA). To establish a PCa xenograft model, $2 \times 10^{6}$ PC-3 cells stably overexpressing PIK3CD-L or PIK3CD-S were subcutaneously injected into the left flank of NOD-SCID mice. Tumour xenograft growth was measured with calipers and the volume was determined as $1 / 2 \times$ length $\times$ width $^{2}$. Mice were randomized into groups once the average tumour size reached $\sim 200 \mathrm{~mm}^{3}$ and treated with vehicle (phosphate-buffered saline) or CAL-101 (50 $\left.\mathrm{mg} \mathrm{kg}^{-1}\right)$ through daily i.p. injections. After 30 days, mice were euthanized and the dissected xenografts were photographed and weighed using a blinded design.

To establish the PCa metastasis model, $1 \times 10^{6}$ PC-3 cells stably overexpressing PIK3CD-L or PIK3CD-S were injected into the tail vein of NOD-SCID mice. The mice were then treated with vehicle or CAL-101 $\left(50 \mathrm{mg} \mathrm{kg}^{-1}\right)$ via i.p. injections, 3 times a week. After 8 weeks, lungs of mice were collected and stained with India ink and bleached with Fekete's solution (70\% ethanol, 3.7\% formaldehyde, $0.75 \mathrm{M}$ glacial acetic acid). India ink-stained lungs were photographed and lung metastases were quantified using the NIH ImageJ program ${ }^{68}$. 
Purification of His-tagged PI3K $\delta$ protein. PC-3 cells stably overexpressing PIK3CD-L or PIK3CD-S were maintained in DMEM (Life Technologies) supplemented with $10 \%$ FBS. After growing the cells for $24 \mathrm{~h}$, cell extracts were prepared and His-tagged PI3K $\delta$ protein was purified using a column HisPur Ni-NTA purification kit (Pierce Biotechnology, Rockford, IL, USA). Briefly, cell lysates were mixed with Ni-NTA resin and incubated at room temperature for $30 \mathrm{~min}$. After incubation, the resin was washed with wash buffer ( $25 \mathrm{mM}$ imidazole, $\mathrm{pH}$ 7.4) and applied to a HisPur Ni-NTA spin column, centrifuged and wash buffer eluate discarded after centrifugations. His-tagged proteins were eluted from the resin by adding one-resin-bed volume of elution buffer ( $250 \mathrm{mM}$ imidazole, $\mathrm{pH}$ 7.4). The purified PI $3 \mathrm{~K} \delta$-His protein was mixed with $2 \times$ Laemmli sample buffer, boiled and analysed by immunoblotting.

Co-IP of PI3K $\mathbf{\delta} / \mathbf{p 8 5}$ complex. Plasmids pcDNA3.1-PIK3CD-S/V5-His (or pcDNA3.1-PIK3CD-L/V5-His) and pSV-p85 $\alpha$ (Addgene, Cambridge, MA, USA) were co-transfected into PC-3 cells. After growing the cells for $48 \mathrm{~h}$, the co-transfected cells were collected and cells were lysed with RIPA lysis buffer (Santa Cruz Biotechnology). The cell lysates were then subjected to Co-IP assays with anti-His antibody (ab18184, Abcam) and immobilized on protein G-Sepharose beads (Thermo Scientific, Waltham, MA, USA). Cell lysates and precipitates were subjected to western blotting, and visualized by enhanced chemiluminescence system (Thermo Scientific, Waltham, MA, USA).

In vitro assay of PI3Kס activity. PI3K $\delta$ activity was evaluated with a $\mathrm{PI} 3 \mathrm{~K}$ activity/inhibitor assay kit (Millipore, Billerica, MA, USA) according to the manufacturer's instructions. Briefly, purified His-tagged PI3K $\delta$-L or PI3K $\delta$-S isoform was pretreated with the PI3K $\delta$ inhibitor $(100 \mathrm{nM}$ of wortmannin or $100 \mathrm{nM}$ of CAL-101) or vehicle in 96-well plates for $10 \mathrm{~min}$ and subjected to a competitive ELISA. PIP2 substrate and kinase reaction buffer were added to the pretreated His-tagged PI $3 \mathrm{~K} \delta$-L or $\mathrm{PI} 3 \mathrm{~K} \delta$-S isoform and incubated at room temperature for $1 \mathrm{~h}$. After incubation, biotinylated PIP3 and GST-GRP1 working solutions were added to the wells and the reaction samples were further incubated at room temperature for $1 \mathrm{~h}$. Plates were washed three times with $1 \times$ Tris-buffered saline with Tween-20 (150 mM NaCl, $0.1 \%$ Tween-20, $50 \mathrm{mM}$ Tris-Cl, pH 7.5) and incubated with streptavidin-horseradish peroxidase conjugate $\left(1.25 \mathrm{mg} \mathrm{ml}^{-1}\right)$ at room temperature for $1 \mathrm{~h}$. After incubation, plates were washed three times and incubated with $100 \mu \mathrm{l}$ of TMB $\left(3,3^{\prime}, 5,5^{\prime}\right.$-tetramethylbenzidine, $\left.1 \mathrm{mg} \mathrm{ml}^{-1}\right)$ substrate solution at room temperature for 5-20 min. Reactions were stopped by adding $100 \mu \mathrm{l}$ of stop solution and plates read at $450 \mathrm{~nm}$. The colourimetric signal was inversely proportional to the amount of PIP 3 produced by PI3K activity and the relative amount of PIP3 produced was determined with a standard curve.

Analysis of in-frame and out-of-frame exon skipping events. A total of 4,253 significant differentially expressed exons ( $2 \%$ FDR) were identified. Using a 1.5 -fold cutoff, the number of differentially expressed exons (that is, exon skipping events) was narrowed down to 3,112 (corresponding to 2,520 DS genes in AA PCa versus EA PCa). The reference coordinates for the Affymetrix probe sets used to identify these skipped exons were cross-referenced with the Ensembl database, release GRCh37, resulting in final set of 2,517 well-curated, differentially expressed exons that corresponded to 1,484 DS genes (Supplementary Data 4). Based on the exon size and the modelled effect, the exon skipping events were classified as inframe or frame-shift (Supplementary Data 4). A total of 1376 genes were included in this analysis. The distribution of in-frame and frameshift events was then compared between the EA and AA groups. We grouped the observations based on presence of at least one frameshift per gene. Notably, we observed significantly higher proportion of alternatively spliced genes without a frameshift in the AA group (34\% in AA versus $27 \%$ in EA, $P<0.005$, Fisher's exact test).

Metrics for assessing reliability of global analysis of DS events. Affymetrix Exon GeneChip analysis has revealed $\sim 2,500$ differential splicing events between AA and EA PCa. While we can only draw firm conclusions on a subset of differential splicing patterns that were validated by a second approach (that is, RTPCR), the following metrics have been provided to allow evaluation of the overall reliability of Exon Genechip results:

(1) Validation success rate. Eight of 9 genes (89\%) identified by Exon GeneChip analysis to undergo differential RNA splicing between AA PCa and EA PCa were successfully validated by quantitative RT-PCR. The only gene that did not validate was EPHA1. In addition, 2 out of 2 genes (100\%), defined not to exhibit differential RNA splicing (GSK3A and $A T M$ ), were successfully validated by quantitative RT-PCR. Aggregate success rate was $91 \%(10 / 11)$.

(2) $P$ values. The $P$ values ranged from $1 \times 10^{-3}$ to $1 \times 10^{-15}$ for the 9 genes identified by Exon GeneChip analysis to undergo differential RNA splicing between AA and EA PCa specimens $\left(P=6 \times 10^{-4}\right.$ for ATM). In comparison, $P$ values for all genes identified by Exon GeneChip analysis to exhibit differential RNA splicing ranged from $3 \times 10^{-3}$ to $1 \times 10^{-20}$ (see Supplementary Data 1). Hence, $P$ values of genes chosen for RT-PCR validation were representative of the entire range of $P$ values associated with the complete set of $\sim 2,500$ genes identified by Exon ChipGene analysis.
(3) Power calculations. Based on the number of PCa patient samples interrogated by our Affymetrix Exon GeneChip arrays $(n=15$ to 20 patients per gene per race) and a computed s.d. $=0.4$, our findings correspond to $>85 \%$ power to distinguish 1.5 -fold changes at $P<0.01$.

Estimation of PIK3CD isoform expression. Expression of the short and long isoforms of PIK3CD was determined using the method IsoformEx ${ }^{69}$. Briefly, isoform expression was estimated through the minimizing a weighted nonnegative least squares problem based on the exon expression. For the purpose of this analysis, the novel 'short isoform' was defined as any transcript missing exon 20 but having exons 19 and 21 concatenated; and the 'long isoforms' were defined as any transcripts with exons 19, 20 and 21 concatenated. Raw data for breast invasive carcinoma (BRCA), prostate adenocarcinoma (PRAD) and colorectal adenocarcinoma (COAD) were obtained from TCGA RNA-sequencing exon expression (https://tcga-data.nci.nih.gov/tcga/, accessed 22 January 2016). The ratio of short to long isoforms was calculated for survival analysis.

Survival analysis. Disease-free survival data for BRCA, COAD and PRAD were obtained from TCGA clinical data (https://tcga-data.nci.nih.gov/tcga/, accessed 22 January 2016). Patients who did not have a relapse event during the study were considered as censored. The expression values of the short and long isoforms as well as the interaction term were used as predictors to fit the Cox proportional hazards regression model under L2-regularization, where the disease-free survival is the response variable. For each patient, a prognosis index score was computed from the Cox proportional hazards model ${ }^{70}$. Briefly, the patients were dichotomized into high- and low-risk groups according to the relapsed versus relapse-free ratio. The $\log$-rank $P$ value was then calculated to assess the statistically significant difference between the Kaplan-Meier curves of the high- versus low-risk groups.

Data availability. The authors declare that all data supporting the findings of this study are available within the article and its Supplementary Information files or from the corresponding author on reasonable request. Sequences of PIK3CD-S and PIK3CD- $L$ were deposited to GenBank (accession numbers KU612116 and KU612117).

\section{References}

1. Jemal, A. et al. Cancer statistics, 2007. CA Cancer. J. Clin. 57, 43-66 (2007).

2. Reddy, S., Shapiro, M., Morton, Jr R. \& Brawley, O. W. Prostate cancer in black and white Americans. Cancer Metastasis. Rev. 22, 83-86 (2003).

3. Powell, I. J. Epidemiology and pathophysiology of prostate cancer in AfricanAmerican men. J. Urol. 177, 444-449 (2007).

4. Jones, B. A. et al. Explaining the race difference in prostate cancer stage at diagnosis. Cancer Epidemiol. Biomarkers Prev. 17, 2825-2834 (2008).

5. Evans, S., Metcalfe, C., Ibrahim, F., Persad, R. \& Ben-Shlomo, Y. Investigating Black-White differences in prostate cancer prognosis: a systematic review and meta-analysis. Int. J. Cancer. 123, 430-435 (2008).

6. Kittles, R. A. et al. A common nonsense mutation in EphB2 is associated with prostate cancer risk in African American men with a positive family history. J. Med. Genet. 43, 507-511 (2006).

7. Powell, I. J. \& Bollig-Fischer, A. Minireview: the molecular and genomic basis for prostate cancer health disparities. Mol. Endocrinol. 27, 879-891 (2013).

8. Wallace, T. A. et al. Tumor immunobiological differences in prostate cancer between African-American and European-American men. Cancer Res. 68, 927-936 (2008).

9. Wang, B. D. et al. Identification and functional validation of reciprocal microRNA-mRNA pairings in African American prostate cancer disparities. Clin. Cancer Res. 21, 4970-4984 (2015).

10. Wang, B. D. et al. Androgen receptor-target genes in african american prostate cancer disparities. Prostate Cancer 2013, 763569 (2013).

11. Pan, Q., Shai, O., Lee, L. J., Frey, B. J. \& Blencowe, B. J. Deep surveying of alternative splicing complexity in the human transcriptome by high-throughput sequencing. Nat. Genet. 40, 1413-1415 (2008).

12. David, C. J. \& Manley, J. L. Alternative pre-mRNA splicing regulation in cancer: pathways and programs unhinged. Genes Dev. 24, 2343-2364 (2010).

13. Chen, J. \& Weiss, W. A. Alternative splicing in cancer: implications for biology and therapy. Oncogene 34, 1-14 (2015).

14. Wang, J. et al. Pleiotropic biological activities of alternatively spliced TMPRSS2/ ERG fusion gene transcripts. Cancer Res. 68, 8516-8524 (2008).

15. Mercatante, D. R., Mohler, J. L. \& Kole, R. Cellular response to an antisensemediated shift of Bcl-x pre-mRNA splicing and antineoplastic agents. J. Biol. Chem. 277, 49374-49382 (2002).

16. Rajan, P., Elliott, D. J., Robson, C. N. \& Leung, H. Y. Alternative splicing and biological heterogeneity in prostate cancer. Nat. Rev. Urol. 6, 454-460 (2009).

17. Akgul, C., Moulding, D. A. \& Edwards, S. W. Alternative splicing of Bcl-2related genes: functional consequences and potential therapeutic applications. Cell. Mol. Life Sci. 61, 2189-2199 (2004).

18. Sun, A. et al. Androgen receptor-dependent regulation of $\mathrm{Bcl}-\mathrm{xL}$ expression: Implication in prostate cancer progression. Prostate 68, 453-461 (2008). 
19. Bauman, J. A., Li, S. D., Yang, A., Huang, L. \& Kole, R. Anti-tumor activity of splice-switching oligonucleotides. Nucleic Acids Res. 38, 8348-8356 (2010).

20. Oltean, S. et al. Alternative inclusion of fibroblast growth factor receptor 2 exon IIIc in Dunning prostate tumors reveals unexpected epithelial mesenchymal plasticity. Proc. Natl Acad. Sci. USA 103, 14116-14121 (2006).

21. Demichelis, F. et al. TMPRSS2:ERG gene fusion associated with lethal prostate cancer in a watchful waiting cohort. Oncogene 26, 4596-4599 (2007).

22. $\mathrm{Hu}, \mathrm{Y}$. et al. Delineation of TMPRSS2-ERG splice variants in prostate cancer. Clin. Cancer Res. 14, 4719-4725 (2008).

23. Heinlein, C. A. \& Chang, C. Androgen receptor in prostate cancer. Endocr. Rev. 25, 276-308 (2004).

24. Antonarakis, E. S. et al. AR-V7 and resistance to enzalutamide and abiraterone in prostate cancer. N. Engl. J. Med. 371, 1028-1038 (2014).

25. $\mathrm{Hu}, \mathrm{R}$. et al. Ligand-independent androgen receptor variants derived from splicing of cryptic exons signify hormone-refractory prostate cancer. Cancer Res. 69, 16-22 (2009).

26. Fan, W., Stirewalt, D. L., Radich, J. P. \& Zhao, L. Comparison of two methods for detecting alternative splice variants using genechip $((\mathrm{R}))$ exon arrays. Int. J. Biomed. Sci. 7, 172-180 (2011).

27. Cancer Genome Atlas Research Network. Comprehensive genomic characterization defines human glioblastoma genes and core pathways. Nature 455, 1061-1068 (2008).

28. Ding, L. et al. Somatic mutations affect key pathways in lung adenocarcinoma. Nature 455, 1069-1075 (2008).

29. Cancer Genome Atlas Research Network. Integrated genomic analyses of ovarian carcinoma. Nature 474, 609-615 (2012).

30. Boller, D. et al. Targeting the phosphoinositide 3-kinase isoform p110delta impairs growth and survival in neuroblastoma cells. Clin. Cancer Res. 14, 1172-1181 (2008).

31. Jiang, X., Chen, S., Asara, J. M. \& Balk, S. P. Phosphoinositide 3-kinase pathway activation in phosphate and tensin homolog (PTEN)-deficient prostate cancer cells is independent of receptor tyrosine kinases and mediated by the p110beta and p110delta catalytic subunits. J. Biol. Chem. 285, 14980-14989 (2010).

32. Knobbe, C. B. \& Reifenberger, G. Genetic alterations and aberrant expression of genes related to the phosphatidyl-inositol-3'-kinase/protein kinase B (Akt) signal transduction pathway in glioblastomas. Brain Pathol. 13, 507-518 (2003).

33. Tzenaki, N. et al. High levels of p110delta PI3K expression in solid tumor cells suppress PTEN activity, generating cellular sensitivity to p110delta inhibitors through PTEN activation. FASEB J. 26, 2498-2508 (2012).

34. Glickman, M., Malek, R. L., Kwitek-Black, A. E., Jacob, H. J. \& Lee, N. H. Molecular cloning, tissue-specific expression, and chromosomal localization of a novel nerve growth factor-regulated G-protein- coupled receptor, nrg-1. Mol. Cell Neurosci. 14, 141-152 (1999).

35. Korenchuk, S. et al. VCaP, a cell-based model system of human prostate cancer. In Vivo 15, 163-168 (2001).

36. Navone, N. M. et al. Establishment of two human prostate cancer cell lines derived from a single bone metastasis. Clin. Cancer Res. 3, 2493-2500 (1997).

37. Herman, S. E. et al. Phosphatidylinositol 3-kinase-delta inhibitor CAL-101 shows promising preclinical activity in chronic lymphocytic leukemia by antagonizing intrinsic and extrinsic cellular survival signals. Blood 116, 2078-2088 (2010).

38. Lannutti, B. J. et al. CAL-101, a p110delta selective phosphatidylinositol-3kinase inhibitor for the treatment of B-cell malignancies, inhibits PI3K signaling and cellular viability. Blood 117, 591-594 (2011).

39. Fruman, D. A. \& Rommel, C. PI3Kdelta inhibitors in cancer: rationale and serendipity merge in the clinic. Cancer Discov. 1, 562-572 (2011).

40. Yang, J. et al. Pathological axonal death through a MAPK cascade that triggers a local energy deficit. Cell 160, 161-176 (2015).

41. Berndt, A. et al. The p110 delta structure: mechanisms for selectivity and potency of new PI(3)K inhibitors. Nat. Chem. Biol. 6, 117-124 (2010)

42. Geering, B., Cutillas, P. R., Nock, G., Gharbi, S. I. \& Vanhaesebroeck, B. Class IA phosphoinositide 3-kinases are obligate p85-p110 heterodimers. Proc. Natl Acad. Sci. USA 104, 7809-7814 (2007).

43. Fry, M. J. et al. Purification and characterization of a phosphatidylinositol 3-kinase complex from bovine brain by using phosphopeptide affinity columns. Biochem. J. 288, 383-393 (1992).

44. Woscholski, R., Dhand, R., Fry, M. J., Waterfield, M. D. \& Parker, P. J. Biochemical characterization of the free catalytic p110 alpha and the complexed heterodimeric p110 alpha.p85 alpha forms of the mammalian phosphatidylinositol 3-kinase. J. Biol. Chem. 269, 25067-25072 (1994).

45. Fransson, S. et al. p37delta is a new isoform of PI3K p110delta that increases cell proliferation and is overexpressed in tumors. Oncogene 31, 3277-3286 (2012).

46. Arcaro, A. \& Wymann, M. P. Wortmannin is a potent phosphatidylinositol 3-kinase inhibitor: the role of phosphatidylinositol 3,4,5-trisphosphate in neutrophil responses. Biochem. J. 296 Pt 2 297-301 (1993).
47. Yang, Y. et al. Dysregulation of microRNA-212 promotes castration resistance via hnRNPH1-mediated regulation of $A R$ and AR-V7: implications for racial disparity of prostate cancer. Clin. Cancer Res. 22, 1744-1756 (2016).

48. Modrek, B., Resch, A., Grasso, C. \& Lee, C. Genome-wide detection of alternative splicing in expressed sequences of human genes. Nucleic Acids Res. 29, 2850-2859 (2001).

49. Sorek, R., Shamir, R. \& Ast, G. How prevalent is functional alternative splicing in the human genome? Trends Genet. 20, 68-71 (2004).

50. Blencowe, B. J. Alternative splicing: new insights from global analyses. Cell 126, 37-47 (2006).

51. Courtney, K. D., Corcoran, R. B. \& Engelman, J. A. The PI3K pathway as drug target in human cancer. J. Clin. Oncol. 28, 1075-1083 (2010).

52. Ciraolo, E., Morello, F. \& Hirsch, E. Present and future of PI3K pathway inhibition in cancer: perspectives and limitations. Curr. Med. Chem. 18, 2674-2685 (2011).

53. Clayton, E. et al. A crucial role for the p110delta subunit of phosphatidylinositol 3-kinase in B cell development and activation. J. Exp. Med. 196, 753-763 (2002).

54. Jou, S. T. et al. Essential, nonredundant role for the phosphoinositide 3-kinase p110delta in signaling by the B-cell receptor complex. Mol. Cell. Biol. 22, 8580-8591 (2002).

55. Castillo, J. J., Furman, M. \& Winer, E. S. CAL-101: a phosphatidylinositol-3kinase p110-delta inhibitor for the treatment of lymphoid malignancies. Expert Opin. Investig. Drugs 21, 15-22 (2012).

56. Nakazawa, M., Antonarakis, E. S. \& Luo, J. Androgen receptor splice variants in the era of enzalutamide and abiraterone. Horm. Cancer 5, 265-273 (2014).

57. Shi, H. et al. Acquired resistance and clonal evolution in melanoma during BRAF inhibitor therapy. Cancer Discov. 4, 80-93 (2014).

58. Berndt, A. et al. The p110delta structure: mechanisms for selectivity and potency of new PI(3)K inhibitors. Nat. Chem. Biol. 6, 117-124 (2010).

59. Gopal, A. K. et al. PI3Kdelta inhibition by idelalisib in patients with relapsed indolent lymphoma. N. Engl. J. Med. 370, 1008-1018 (2014).

60. Brown, J. R. et al. Idelalisib, an inhibitor of phosphatidylinositol 3-kinase p110delta, for relapsed/refractory chronic lymphocytic leukemia. Blood 123, 3390-3397 (2014).

61. Shah, A. \& Mangaonkar, A. Idelalisib: a novel PI3Kdelta inhibitor for chronic lymphocytic leukemia. Ann. Pharmacother. 49, 1162-1170 (2015).

62. Vanhaesebroeck, B., Guillermet-Guibert, J., Graupera, M. \& Bilanges, B. The emerging mechanisms of isoform-specific PI3K signalling. Nat. Rev. Mol. Cell Biol. 11, 329-341 (2010).

63. Yu, J. et al. Regulation of the p85/p110 phosphatidylinositol 3 '-kinase: stabilization and inhibition of the p110alpha catalytic subunit by the p85 regulatory subunit. Mol. Cell Biol. 18, 1379-1387 (1998).

64. Chan, T. O. et al. Small GTPases and tyrosine kinases coregulate a molecular switch in the phosphoinositide 3-kinase regulatory subunit. Cancer Cell 1, 181-191 (2002).

65. Jaiswal, B. S. et al. Somatic mutations in p85alpha promote tumorigenesis through class IA PI3K activation. Cancer Cell 16, 463-474 (2009).

66. Amzel, L. M. et al. Structural comparisons of class I phosphoinositide 3-kinases Nat. Rev. Cancer 8, 665-669 (2008).

67. Benjamini, Y. \& Hochberg, Y. Controlling the false discovery rate: a practical and powerful approach to multiple testing. J. R. Statist. Soc. 57, 289-300 (1995)

68. Fukuda, H. et al. Host-derived MMP-13 exhibits a protective role in lung metastasis of melanoma cells by local endostatin production. Br. J. Cancer 105, 1615-1624 (2011).

69. Kim, H., Bi, Y., Pal, S., Gupta, R. \& Davuluri, R. V. IsoformEx: isoform level gene expression estimation using weighted non-negative least squares from mRNA-Seq data. BMC Bioinformatics 12, 305 (2011).

70. Huang, S., Yee, C., Ching, T., Yu, H. \& Garmire, L. X. A novel model to combine clinical and pathway-based transcriptomic information for the prognosis prediction of breast cancer. PLoS Comput. Biol. 10, e1003851 (2014).

\section{Acknowledgements}

This research was supported by ACS IRG-08-091-01 Grant (to B.-D.W.), NCI Grant U01CA11637-05S2 (to S.R.P.) and Affymetrix Collaborations in Cancer Research Award, DOD Grant PCRP:PC121975 and NCI Grant CA204806 (to N.H.L.)

\section{Author contributions}

B.-D.W. and N.H.L. conceived and designed the study. B.-D.W. and J.O. performed the microarray experiments, tissue sample analysis and in vitro assays. B.-D.W., V.P., K.C. and S.H. were involved in animal work. R.A. collected clinical samples for the study. B.-D.W., N.H.L., A.H., T.C., L.G. and M.A.G.-B. carried out genomic data analysis. B.D.W. and S.K. performed three-dimensional (3D) modelling of protein isoforms. S.R.P. initiated and organized clinical sample collection at beginning of study and provided input in the Discussion section. B.-D.W., J.A.F. and N.H.L. wrote the manuscript with input from all authors. N.H.L. supervised the project. 


\section{Additional information}

Supplementary Information accompanies this paper at http://www.nature.com/ naturecommunications

Competing interests: The authors declare no competing financial interests.

Reprints and permission information is available online at http://npg.nature.com/ reprintsandpermissions/

How to cite this article: Wang, B.-D. et al. Alternative splicing promotes tumour aggressiveness and drug resistance in African American prostate cancer. Nat. Commun. 8, 15921 doi: 10.1038/ncomms15921 (2017).

Publisher's note: Springer Nature remains neutral with regard to jurisdictional claims in published maps and institutional affiliations. cc (i) Open Access This article is licensed under a Creative Commons Attribution 4.0 International License, which permits use, sharing, adaptation, distribution and reproduction in any medium or format, as long as you give appropriate credit to the original author(s) and the source, provide a link to the Creative Commons license, and indicate if changes were made. The images or other third party material in this article are included in the article's Creative Commons license, unless indicated otherwise in a credit line to the material. If material is not included in the article's Creative Commons license and your intended use is not permitted by statutory regulation or exceeds the permitted use, you will need to obtain permission directly from the copyright holder. To view a copy of this license, visit http://creativecommons.org/ licenses/by/4.0/

(C) The Author(s) 2017 


\title{
Corrigendum: Alternative splicing promotes tumour aggressiveness and drug resistance in African American prostate cancer
}

\author{
Bi-Dar Wang, Kristin Ceniccola, SuJin Hwang, Ramez Andrawis, Anelia Horvath, Jennifer A. Freedman, \\ Jacqueline Olender, Stefan Knapp, Travers Ching, Lana Garmire, Vyomesh Patel, Mariano A. Garcia-Blanco, \\ Steven R. Patierno \& Norman H. Lee
}

Nature Communications 8:15921 doi: 10.1038/ncomms15921 (2017); Published 30 Jun 2017; Updated 27 Sep 2017

Contributions of the author Steven R. Patierno are not fully acknowledged in this Article. The Author Contributions section should have included the following:

'S.R.P. was involved in the co-conception of the study, and was co-principal investigator of the initial portion of this study by obtaining NCI Center to Reduce Cancer Health Disparities grant supplement U01CA11637-05S2 (to S.R.P.) that supported initial experiments towards differential alternative splicing events, oncogenic splicing pathway, validation of differential alternative splicing and selective knockdown of PIK3CD L and S variants.'

(c) Open Access This article is licensed under a Creative Commons Attribution 4.0 International License, which permits use, sharing, adaptation, distribution and reproduction in any medium or format, as long as you give appropriate credit to the original author(s) and the source, provide a link to the Creative Commons license, and indicate if changes were made. The images or other third party material in this article are included in the article's Creative Commons license, unless indicated otherwise in a credit line to the material. If material is not included in the article's Creative Commons license and your intended use is not permitted by statutory regulation or exceeds the permitted use, you will need to obtain permission directly from the copyright holder. To view a copy of this license, visit http://creativecommons.org/licenses/by/4.0/

(C) The Author(s) 2017 